\title{
Nucleon-Nucleon scattering from dispersion relations: next-to-next-to-leading order study
}

\author{
J. A. Oller \\ Departamento de Física. Universidad de Murcia. \\ E-30071 Murcia, Spain. \\ oller@um.es
}

\begin{abstract}
We study nucleon-nucleon $(N N)$ scattering by applying the $N / D$ method in chiral perturbation theory up to next-to-next-to-leading (NNLO) order in the calculation of the imaginary part of the $N N$ partial-wave amplitudes along the left-hand-cut, which is the dynamical input for this approach. A quite good reproduction of the Nijmegen partial-wave analysis phase shifts and mixing angles is obtained, which implies a steady improvement in the accurateness achieved by increasing the chiral order in the calculation of the dynamical input. A power counting for the subtraction constants is established, which is appropriate for those subtractions attached to both the left- and right-hand cuts. We discuss that it is not necessary to modify the $N N$ chiral potential at NNLO to agree with data, but instead one should perform the iteration of two-nucleon intermediate states to finally achieve analytic and unitarity $N N$ partial-wave amplitudes in a well-defined way. We also confirm at NNLO the long-range correlations between the $N N S$-wave effective ranges and scattering lengths, when employing only once-subtracted dispersion relations, that holds up to around $10 \%$ when compared with experimental values.
\end{abstract}




\section{Introduction}

Chiral Perturbation Theory (ChPT) is the effective field theory of QCD at low energies [1,2]. Its paradigmatic application is the purely mesonic sector in $S U(2) 11$ Its extension to the one-baryon sector presents some complications due to the large nucleon mass that does not vanish in the chiral limit [13, 14], which posed interesting problems to the theory 2 For reviews on ChPT on these topics see e.g. [18, 22].

The extension of ChPT to systems with a larger baryonic number was considered in Ref. [23, where the chiral counting is applied to the calculation of the multi-nucleon potential. In these cases one also has to face the problem associated with the infrared enhancement associated with the small nucleon kinetic energies, which requires to resum the infinite string of diagrams due to the iteration of intermediate multi-nucleon states. The extension of the chiral power counting to finite density system, including the contributions of multi-nucleon reducible diagrams, is given in Ref. [24]. For related reviews see e.g. [25 29].

The application of the set up of Ref. [23] to nucleon-nucleon $(N N)$ scattering has been phenomenologically successful [30 33]. However, the sensitivity of the results on the values of the cutoff taken to solve the associated Lippmann-Schwinger equation for the iteration of two-nucleon intermediate states has given rise to a flurry of publications, whose fair and comprehensive consideration is beyond this introduction. For more detailed accounts on this respect the reader is referred to [25, 26, 34, 40].

We continue here the application of the $N / D$ method [41] to $N N$ scattering extending the previous work of Refs. [42 44]. For this method the dynamical input is not the $N N$ potential but the discontinuity of a $N N$ partial-wave amplitude along the left-hand-cut (LHC), which is denoted in the following by $2 i \Delta(A)$. Here $A$ is the center of mass (c.m.) three-momentum squared of a $N N$ state. In other words, $\Delta(A)$ is the imaginary part of a $N N$ partial-wave amplitude along the LHC, that extends for real $A$ with $A<-M_{\pi}^{2} / 4$, being $M_{\pi}$ the pion $(\pi)$ mass. The function $\Delta(A)$ is due to the multi-exchange of pions driving the finite-range nuclear forces, while in a low-energy effective field theory the short-range nuclear forces are accounted for by local interactions of zero range that do not contribute to $\Delta(A)$ for finite $A$. The two-nucleon irreducible contributions to $\Delta(A)$ are amenable to a straightforward ChPT expansion, in much the same way as discussed in Ref. [23] for the calculation of the chiral $N N$ potential. However, $\Delta(A)$ has also contributions from two-nucleon reducible diagrams but, as explained in Ref. [44], these contributions require to cut all the pion lines simultaneously when iterating one-pion exchange (OPE). In this way, when including an extra $N N$ intermediate state in the iteration of the unitarity two-nucleon diagrams their contribution to $\Delta(A)$ starts further away in the LHC. It then results that the $n$th iteration of two-nucleon intermediate states, which at least requires $n+1$ OPE ladders, gives contribution to $\Delta(A)$ only for $A<-(n+1)^{2} M_{\pi}^{2} / 4$. This makes that its relevance for physical values of $A(A \geq 0)$ in the lowenergy region clearly dismisses with increasing $n$. As a result, because of the chiral expansion together with this other effect that numerically suppresses the proliferation of two-nucleon reducible diagrams in the calculation of $\Delta(A)$, one can determine this function reliably in ChPT 3

In Refs. [42,43] the $N / D$ method was solved with $\Delta(A)$ calculated at leading order (LO) from OPE, while in Ref. [44 the NLO contributions to $\Delta(A)$ were also included. These contributions comprise two-nucleon irreducible two-pion exchange and once-iterated OPE, whose sum gives the leading two-pion

\footnotetext{
${ }^{1}$ Which even presents one corner of concern due to the enhanced role of the right-hand-cut in the isoscalar scalar pion-pion scattering [3], with an important impact as well in the pion-nucleon $(\pi N)$ sector [10-12].

${ }^{2} \mathrm{~A}$ faster stabilization of the chiral series in this case has been recently accomplished 111215 by combining the covariant formalism of the Extended on Mass Shell Regularization Scheme (EOMS) 16] with the explicit inclusion of the $\Delta(1232)$ in the $\delta$-counting [17].

${ }^{3}$ Notice that the suppression of the iteration of two-nucleon reducible diagrams only occurs for $\Delta(A)$, and it does not occur to any other "component" of a $N N$ partial wave amplitude.
} 
exchange (TPE). Reference [44] obtained a clear improvement in the reproduction of the phase shifts and mixing angles given by the Nijmegen partial-wave analysis (PWA) [45] as compared with the LO study, so that a global and rather good agreement is achieved at NLO. We want to give one step forward and consider here the next-to-next-to-leading (NNLO) contributions to $\Delta(A)$, which are given by the imaginary part along the LHC of the two-nucleon irreducible TPE diagrams with a NLO $\pi N$ vertex in Heavy-Baryon ChPT (HBChPT) [46. We see that the chiral expansion within our approach is well behaved, so that there is a steady improvement in the reproduction of the Nijmegen PWA results when passing from LO to NLO and then to NNLO, where a quite good reproduction of the Nijmegen PWA is finally obtained. This is accomplished in a progressive and smooth way, without violent variations in the results obtained at every order 4 In addition, we deal with convergent integrals by taking enough number of subtractions so that the above referred regulator dependence that arises when solving the LippmannSchwinger equation with a chiral $N N$ potential is avoided by construction in our approach. An interesting outcome from our study is that we corroborate the long-range correlations between the effective range and scattering length for each of the $N N S$ waves, ${ }^{1} S_{0}$ and ${ }^{3} S_{1}$, when only the corresponding scattering length is taken as experimental input. These correlations, first noticed in Ref. [34], were also obtained in the NLO $N / D$ study of Ref. [44], and within our approach they are deduced solely from basic principles of $N N$ partial-wave amplitudes, namely, chiral symmetry, unitarity and analyticity. They are typically fulfilled at the level of around a $10 \%$ when comparing with the experimental values for the effective ranges. We should say that we can proceed further and include more subtractions, so that we can implement within our formalism the exact values of the effective ranges, something not possible in the tight scheme of Ref. [34].

Regarding the subtraction constants we elaborate below a chiral power counting for them, by taking into account the change in their values due to variations in the subtraction point. We show that at NLO and NNLO in the calculation of $\Delta(A)$ one properly takes twice-subtracted dispersion relations (DRs). Nevertheless, on top of this criterion we impose that one should obtain the proper threshold behavior for higher partial waves, as well as having meaningful solutions of the integral equations (IEs) that result from the corresponding DRs 5 These two requirements often imply the necessity of taking more than two subtractions in the corresponding DRs relations. Regarding the number of subtractions used to guarantee the threshold behavior for higher partial waves we use here the formalism developed in Ref. [4], so that partial waves with orbital angular momentum $\ell \geq 1$ and mixing partial waves with total angular momentum $J \geq 1$ vanish at threshold as $A^{\ell}$ and $A^{J}$, respectively. This requires to take at least $\ell$ or $J$ subtractions, in order, with $\ell-1$ or $J-1$ free parameters, respectively. But at the end, as emphasized in Ref. [44, none or only one of the resulting subtraction constants for a given partial wave with $\ell>1$ (or $J>1$ for a mixing wave) is necessary to reproduce data. This interesting point, which allows to treat easily higher partial waves, is called in Ref. 44 the principle of maximal smoothness.

In our study we have also paid special attention to the issue concerning the impact on the results of the rather large size of the NLO $\pi N$ counterterms, typically denoted by $c_{i}$ [22], which first appear in the calculation of $\Delta(A)$ at NNLO. It is discussed in Ref. [48] that the $\pi N$ monomials, proportional to the $c_{i}$ counterterms, produce a too large contribution to the $N N$ potential at medium and short distances when it is calculated at NNLO in dimensional regularization, which worsens the properties of the chiral expansion. Because of this Ref. [48] argued to better use a cutoff regularization to calculate the NNLO potential, or equivalently, to cut the energy spectral representation of the NNLO $N N$ potential at around

\footnotetext{
${ }^{4}$ This was not the case in previous studies, e.g. in the model calculation of $N N$ scattering by Ref. 47] that uses a modified version of the $N / D$ method by truncating the integrals along the LHC with a sharp cutoff.

${ }^{5}$ By a meaningful solution we mean here a mathematical solution to the IE that does not depend on the the number of points employed and in the arbitrary large extension of the LHC on which they lie when performing the numerical discretization to solve the IE.
} 
the chiral symmetry breaking scale. This last point would be equivalent to truncate the full extent of the LHC in our dispersive integrals. However, it is interesting to remark that we do not need to do that in order to obtain a good reproduction of the Nijmegen PWA when employing $\Delta(A)$ determined up to NNLO. In fact, we observe that the definitive improvement of our results compared with the Born approximation does not arise by modifying the two-nucleon irreducible diagrams at NNLO, but by performing the iteration of two-nucleon unitarity diagrams as required by analyticity and unitarity in a well-defined way.

After this introduction we review the $N / D$ method for coupled and uncoupled partial waves in Sec. 2 , The function $\Delta(A)$, calculated in ChPT up to NNLO, is discussed in Sec. 3, where we also elaborate the chiral power counting for the subtraction constants. Sections 4 to 14 are devoted to discuss the application of the $N / D$ method to the different $N N$ partial waves up to $J=5$. There it is shown that a quite good reproduction of the Nijmegen PWA phase shifts and mixing angles results. In these sections we also compare with the Born approximation for higher partial waves and discuss on the relative importance of the different contributions to $\Delta(A)$. Our concluding remarks are given in Sec. 15, Finally, we discuss in Appendix $\mathrm{A}$ a method to calculate higher order shape parameters of the $N N S$ waves.

\section{The $N / D$ method}

A detailed presentation of the formalism for the $N / D$ method [41] can be found in Ref. [44. Here we only reproduce the main facets of the approach.

\subsection{Uncoupled partial waves}

An uncoupled $N N$ partial wave is written as the quotient of two functions, where the numerator is the function $N(A)$ and the denominator is $D(A)$. Then, one writes

$$
T(A)=\frac{N(A)}{D(A)},
$$

with $T(A)$ the corresponding $N N$ partial wave in the c.m. frame. In the following we use the spectroscopic notation and denote by ${ }^{2 S+1} L_{J}$ the different $N N$ partial waves with $S$ the total spin, $L$ the orbital angular momentum and $J$ the total angular momentum. The point for the splitting of $T(A)$ in two functions is because $N(A)$ has only LHC while $D(A)$ has only right-hand cut (RHC), also called unitarity cut. The following expressions for the discontinuities of the functions $N(A)$ and $D(A)$ along their respective cuts then arise,

$$
\begin{gathered}
\operatorname{Im} D(A)=-\rho(A) N(A), A>0, \\
\operatorname{Im} N(A)=\Delta(A) D(A), A<L .
\end{gathered}
$$

Here $L=-M_{\pi}^{2} / 4$ and it represents the onset of the LHC for $A<L$ due to OPE, and $\rho(A)$ is the phase space factor

$$
\rho(A)=\frac{m \sqrt{A}}{4 \pi},
$$

where $m$ is the nucleon mass. The first of the relations in Eq. (2) is a consequence of elastic unitarity for a $N N$ partial wave, which reads

$$
\operatorname{Im} T(A)=\rho|T(A)|^{2}, A>0 .
$$


In terms of $1 / T(A)$ this can be recast simply as

$$
\operatorname{Im} \frac{1}{T(A)}=-\rho(A), A>0 \text {. }
$$

With this normalization the relation between the $T$ and $S$ matrices is $S(A)=1+2 i \rho(A) T(A)$. The discontinuity of a $N N$ partial wave $T(A)$ along the LHC is given by $2 i \Delta(A)$, which directly implies the second expression in Eq. (2).

Standard DRs for the functions $D(A)$ and $N(A)$ are derived in Ref. [44] under the assumption that the function $D(A)$ does not diverge faster than a polynomial of degree $n_{0}$ for $A \rightarrow \infty$. Then for $n>n_{0}$ one can write [44]

$$
\begin{aligned}
& D(A)=\sum_{i=1}^{n} \delta_{i}(A-C)^{i-1}-\frac{(A-C)^{n}}{\pi} \int_{0}^{\infty} d q^{2} \frac{\rho\left(q^{2}\right) N\left(q^{2}\right)}{\left(q^{2}-A\right)\left(q^{2}-C\right)^{n}}, \\
& N(A)=\sum_{i=1}^{n} \nu_{i}(A-C)^{i-1}+\frac{(A-C)^{n}}{\pi} \int_{-\infty}^{L} d k^{2} \frac{\Delta\left(k^{2}\right) D\left(k^{2}\right)}{\left(k^{2}-A\right)\left(k^{2}-C\right)^{n}}
\end{aligned}
$$

where $C$ is the subtraction point. Notice that the same number of subtractions is taken both in $D(A)$ and $N(A)$. The argument given in Ref. [44 makes use of the fact that $N(A)=T(A) D(A)$ and $T(A)$, because of unitarity, vanish at least as $A^{-1 / 2}$ for $A \rightarrow+\infty$. As a result if $D(A)$ diverges at most as $A^{n_{0}}$ then $N(A)$ does not diverge faster than $A^{n_{0}-1 / 2}$. Here we take into account the Sugawara and Kanazawa theorem [49,50], as a consequence of which any function like $D(A)$ or $N(A)$ with only one cut of infinite extent along the real axis has the same limit for $A \rightarrow \infty$ in any direction of the $A$-complex plane. In addition, it is clear from Eq. (6) and the standard theory of DRs [51, that we can take different values for the corresponding subtraction points for each function separately. Indeed, for many partial waves we will take the subtractions for the function $D(A)$ in two different subtraction points, one at $C=0$ and the other at $C=-M_{\pi}^{2}$. This is motivated by the fact that we impose the normalization

$$
D(0)=1,
$$

which can always be done by dividing simultaneously $D(A)$ and $N(A)$ by a constant without altering their ratio corresponding to $T(A)$, Eq. (II). In this way, one subtraction for $D(A)$ is always taken at $C=0$ in order to guarantee straightforwardly the normalization Eq. (77).

To solve $D(A)$ in terms of the input $\Delta(A)$ and the subtraction constants we substitute in Eq. (6) the expression for $N(A)$ into the DR of $D(A)$, so that we end with the following IE for $D(A)$ with $A<L$,

$$
\begin{aligned}
D(A) & =\sum_{i=1}^{n} \delta_{i}(A-C)^{n-i}-\sum_{i=1}^{n} \nu_{i} \frac{(A-C)^{n}}{\pi} \int_{0}^{\infty} d q^{2} \frac{\rho\left(q^{2}\right)}{\left(q^{2}-A\right)\left(q^{2}-C\right)^{n-i+1}} \\
& +\frac{(A-C)^{n}}{\pi^{2}} \int_{-\infty}^{L} d k^{2} \frac{\Delta\left(k^{2}\right) D\left(k^{2}\right)}{\left(k^{2}-C\right)^{n}} \int_{0}^{\infty} d q^{2} \frac{\rho\left(q^{2}\right)}{\left(q^{2}-A\right)\left(q^{2}-k^{2}\right)} .
\end{aligned}
$$

The key point of the method is to solve this IE numerically which provides the knowledge of $D(A)$ for $A<L$. Once $D(A)$ is known along the LHC we can calculate all the functions $D(A), N(A)$ and $T(A)$ in the whole $A$-complex plane. To obtain $D(A)$ one can use Eq. (8) and for $N(A)$ one has the second of the DRs in Eq. (6). Once $N(A)$ and $D(A)$ are known one can calculate $T(A)$ by applying Eq. (1).

Notice also that the integrations along the RHC in Eq. (8) can be done algebraically in terms of the function

$$
g\left(A, k^{2}\right) \equiv \frac{1}{\pi} \int_{0}^{\infty} d q^{2} \frac{\rho\left(q^{2}\right)}{\left(q^{2}-A\right)\left(q^{2}-k^{2}\right)}=\frac{i m / 4 \pi}{\sqrt{A+i 0^{+}}+\sqrt{k^{2}+i 0^{+}}} .
$$


The term $+i 0$ is necessary for negative $A$ or $k^{2}$, with the prescription $\sqrt{-1 \pm i 0}= \pm i \pi$. We can calculate the other RHC integrals of Eq. (8) with higher powers of the factor $\left(q^{2}-C\right)$ in the denominator by simple differentiation with respect to $C$ of the function $g\left(A, k^{2}\right)$,

$$
\frac{\partial^{p-1} g(A, C)}{\partial C^{p-1}}=\frac{(p-1) !}{\pi} \int_{0}^{\infty} d q^{2} \frac{\rho\left(q^{2}\right)}{\left(q^{2}-A\right)\left(q^{2}-C\right)^{p}}
$$

\subsection{Coupled partial waves}

For the case of the triplet partial waves with total angular momentum $J$ we have the mixing between the partial waves with $\ell=J-1$ and $\ell^{\prime}=J+1$, except for the ${ }^{3} P_{0}$. In this case we denote the different coupled partial waves by $t_{i j}(A)$ with $i, j=1,2$, where 1 labels the lower angular momentum $\ell \equiv \ell_{1}$ and 2 the higher one $\ell^{\prime} \equiv \ell_{2}$. All of them are gathered together in the $2 \times 2$ matrix $T(A)$, in terms of which the $S$-matrix reads

$$
\begin{aligned}
& S(A)=I+2 i \rho(A) T(A)
\end{aligned}
$$

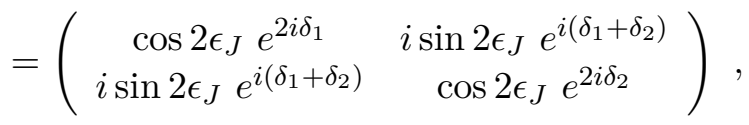

where $I$ is the $2 \times 2$ unit matrix, $\epsilon_{J}$ is the mixing angle, and $\delta_{1}$ and $\delta_{2}$ are the phase shifts for the channels with orbital angular momentum $\ell$ and $\ell^{\prime}$, in this order. Equation (11) corresponds to the Stapp parameterization [52].

Now, the $N / D$ method explained for the uncoupled waves in Sec. (2) is extended to the coupled channel case [43,44] by writing down three $N / D$ equations, one for every $t_{i j}(A)$ [notice that because of time reversal $t_{12}(A)=t_{21}(A)$ ]. The main difference with respect to the uncoupled case is that now the discontinuity along the RHC of the inverse of $t_{i j}(A)$ does not simply correspond to $-\rho(A)$, but it also contains information on the other coupled partial waves. In the following let us employ the notation

$$
\operatorname{Im} \frac{1}{t_{i j}(A)} \equiv-\nu_{i j}(A), A>0 \text {. }
$$

From Eq. (11) it is straightforward to obtain the following expressions for the $\nu_{i j}(A)$ [43, 44],

$$
\begin{aligned}
& \nu_{11}(A)=\rho(A)\left[1-\frac{\frac{1}{2} \sin ^{2} 2 \epsilon_{J}}{1-\cos 2 \epsilon_{J} \cos 2 \delta_{1}}\right]^{-1}, \\
& \nu_{22}(A)=\rho(A)\left[1-\frac{\frac{1}{2} \sin ^{2} 2 \epsilon_{J}}{1-\cos 2 \epsilon_{J} \cos 2 \delta_{2}}\right]^{-1}, \\
& \nu_{12}(A)=2 \rho(A) \frac{\sin \left(\delta_{1}+\delta_{2}\right)}{\sin 2 \epsilon_{J}}
\end{aligned}
$$

In terms of them we have the analogous DRs for $D(A)$ and $N(A)$ of Eq. ([6), but now distinguishing between the different $D_{i j}(A)$ and $N_{i j}(A)$ such that $t_{i j}(A)=N_{i j}(A) / D_{i j}(A)$, and employing $\nu_{i j}(A)$ instead 
of simply $\rho(A)$. The following expressions are obtained [44]:

$$
\begin{aligned}
D_{i j}(A) & =\sum_{p=1}^{n} \delta_{p}^{(i j)}(A-C)^{p-1}-\sum_{p=1}^{n} \nu_{p}^{(i j)} \frac{(A-C)^{n}}{\pi} \int_{0}^{\infty} d q^{2} \frac{\nu_{i j}\left(q^{2}\right)}{\left(q^{2}-A\right)\left(q^{2}-C\right)^{n-p+1}} \\
& +\frac{(A-C)^{n}}{\pi^{2}} \int_{-\infty}^{L} d k^{2} \frac{\Delta_{i j}\left(k^{2}\right) D_{i j}\left(k^{2}\right)}{\left(k^{2}-C\right)^{n}} \int_{0}^{\infty} d q^{2} \frac{\nu_{i j}\left(q^{2}\right)}{\left(q^{2}-A\right)\left(q^{2}-k^{2}\right)}, \\
N_{i j}(A) & =\sum_{p=1}^{n} \nu_{p}^{(i j)}(A-C)^{p-1}+\frac{(A-C)^{n}}{\pi} \int_{-\infty}^{L} d k^{2} \frac{\Delta_{i j}\left(k^{2}\right) D_{i j}\left(k^{2}\right)}{\left(k^{2}-A\right)\left(k^{2}-C\right)^{n}} .
\end{aligned}
$$

Here, we also impose the normalization condition at $A=0$,

$$
D_{i j}(0)=1 \text {. }
$$

Of course, the same remark concerning the subtraction point as done in Sec. 2.1] is also in order here. Namely, we can use different subtraction points for the functions $D_{i j}(A)$ and $N_{i j}(A)$, as well as to use even different subtraction points in the same function, as we will do below for $D_{i j}(A)$.

\section{$2.3 \quad$ Higher partial waves}

An uncoupled $N N$ partial wave with $\ell \geq 1$ should vanish at threshold as $A^{\ell}$. Similarly for a coupled partial wave we have the analogous results but in terms of $\ell_{i j} \equiv\left(\ell_{i}+\ell_{j}\right) / 2$, with $i, j=1$, 2. As discussed in Ref. [44 this threshold behavior is enforced by taken at least $\ell$ or $\ell_{i j}$ subtractions at $C=0$ in the DR

for $N(A)$ in Eq. (6) or Eq. (15), respectively, and setting $\nu_{p}=0\left(\nu_{p}^{(i j)}=0\right)$ for $p=1, \ldots, \ell\left(\ell_{i j}\right)$. In this way we end with the DRs:

$\underline{\text { Uncoupled case : }}$

$$
\begin{aligned}
D(A) & =1+\sum_{p=2}^{\ell} \delta_{p} A^{p-1}+\frac{A^{\ell}}{\pi} \int_{-\infty}^{L} d k^{2} \frac{\Delta\left(k^{2}\right) D\left(k^{2}\right)}{\left(k^{2}\right)^{\ell}} g\left(A, k^{2}\right), \\
N(A) & =\frac{A^{\ell}}{\pi} \int_{-\infty}^{\ell} d k^{2} \frac{\Delta\left(k^{2}\right) D\left(k^{2}\right)}{\left(k^{2}\right)^{\ell}\left(k^{2}-A\right)}, \\
\delta_{p} & =\frac{1}{(p-1) !} D^{(p-1)}(0), p=2,3, \ldots \\
& \text { Coupled case : } \\
D_{i j}(A) & =1+\sum_{p=2}^{\ell_{i j}} \delta_{p}^{(i j)} A(A-C)^{p-2}+\frac{A(A-C)^{\ell_{i j}-1}}{\pi} \int_{-\infty}^{L} d k^{2} \frac{\Delta_{i j}\left(k^{2}\right) D_{i j}\left(k^{2}\right)}{\left(k^{2}\right)^{\ell_{i j}}} g_{i j}\left(A, k^{2}, C ; \ell_{i j-1}\right), \\
N_{i j}(A) & =\frac{A^{\ell_{i j}}}{\pi} \int_{-\infty}^{L} d k^{2} \frac{\Delta_{i j}\left(k^{2}\right) D_{i j}\left(k^{2}\right)}{\left(k^{2}\right)^{\ell_{i j}}\left(k^{2}-A\right)}, \\
\delta_{p}^{(i j)} & =\frac{(-1)^{p}}{C^{p-1}}\left[\sum_{n=0}^{p-2} \frac{(-1)^{n}}{n !} C^{n} D_{i j}^{(n)}(C)-1\right], p=2,3, \ldots
\end{aligned}
$$

where we have denoted the derivative of $D(A)$ of order $n$ by $D^{(n)}(A)$. In addition, we have introduced the function $g_{i j}\left(A, k^{2}, C ; m\right)$ defined as

$$
g_{i j}\left(A, k^{2}, C ; m\right)=\frac{1}{\pi} \int_{0}^{\infty} d q^{2} \frac{\nu_{i j}\left(q^{2}\right)\left(q^{2}\right)^{m}}{\left(q^{2}-A\right)\left(q^{2}-k^{2}\right)\left(q^{2}-C\right)^{m}},
$$


which can be expressed algebraically as a combination of $g(A, B)$ 's, Eq. (9), with different arguments.

Although in this way there is a proliferation of subtraction constants (which are not constrained) in the function $D(A)$ as $\ell\left(\ell_{i j}\right)$ grows, most of them play a negligible role. This is so because $N N$ partial waves with $\ell$ or $\ell_{i j}$ greater than 2 are quite perturbative [44,46]. In practical terms we have found in our NNLO study, as well as in the previous one at NLO [44], that for higher partial waves only $\delta_{\ell}\left(\right.$ or $\delta_{\ell_{i j}}^{(i j)}$ ), if any, is needed to fit data, with the rest of them fixed to zero. Furthermore, no significant improvement in the reproduction of data or in the fitted values is observed by releasing $\delta_{i}$ or $\delta_{i}^{(i j)}$ with $i<\ell$ or $\ell_{i j}$, respectively, so that the fit is stable. This is called in Ref. [44] the principle of maximal smoothness because it implies for the uncoupled case that the derivatives of $D(A)$ at $A=0$ with order $<\ell-1$ are zero, as it follows from Eqs. (17) and (19). Similarly, for the coupled case it implies that $D_{i j}(C)=1$ and $D_{i j}^{(n)}(C)=0$ for $1 \leq n \leq \ell_{i j}-3$, cf. Eqs. (20) and (22). In some cases, it happens that $\delta_{\ell}$ or $\delta_{\ell_{i j}}^{(i j)}$ is also zero and then we say that for this partial wave the subtraction constants have the pure perturbative values.

We further illustrate in this work the perturbative character of $N N$ partial waves with $\ell\left(\ell_{i j}\right) \geq 3$ by comparing the full outcome from the $N / D$ method with the perturbative result corresponding to the leading Born approximation, cf. Sec. 6.1. In this case there is no dependence on any of the subtraction constants $\delta_{p}$ or $\delta_{p}^{(i j)}$ and, indeed, we show below that the results are typically rather similar to the full ones, although the latter reproduce closer the Nijmegen PWA, as one should expect.

\section{The input function $\Delta(A)$}

The discontinuity along the LHC of a NN partial wave, $2 i \Delta(A)$, is taken from the calculation of Ref. [46] in Baryon ChPT (BChPT) up to $\mathcal{O}\left(p^{3}\right)$ or NNLO, which includes OPE plus leading and subleading TPE. At this order $\Delta(A)$ for a given partial wave diverges at most as $\lambda(-A)^{3 / 2}$ for $A \rightarrow-\infty$, with $\lambda$ a constant. As discussed in Ref. [44, when $\lambda<0$ one can have solutions for the integral equation providing $D(A)$ for $A<L$ in the once-subtracted case even with a divergent $\Delta(A)$ for $A \rightarrow-\infty$. However, as we will see below $\lambda$ is not always negative and more subtractions are then required.

\section{1 $\quad$ NLO $\pi N$ counterterms}

At NNLO the function $\Delta(A)$ is sensitive to the NLO $\pi N$ ChPT low-energy constants (LECs) $c_{1}, c_{3}$ and $c_{4}$. We take their values from different works in the literature, that are summarized in Table 1. Within the same reference we distinguish, when appropriate, between those values obtained by fitting phase shifts from the Karlsruhe-Helsinki group (KH) [53] or the George Washington University group (GW) [54].

Reference [55] performs an $\mathcal{O}\left(p^{4}\right)$ HBChPT study of $\pi N$ scattering data. We take its values instead of the ones from the older HBChPT studies at $\mathcal{O}\left(p^{3}\right)$ and $\mathcal{O}\left(p^{4}\right)$ [59]. We include too the values from Lorentz covariant BChPT obtained in Ref. [11 by fitting $\pi N$ phase shifts making use of EOMS at $\mathcal{O}\left(p^{3}\right)$. Furthermore, we show in the table the $c_{i}$ 's obtained in the covariant $\mathcal{O}\left(p^{3}\right)$ BChPT study of Ref. [57] within Infrared Regularization (IR). However, due to the better convergence of the $\pi N$ scattering amplitude in EOMS than in IR [11,12] we give results only for the values obtained within EOMS [11]. The resulting uncertainty band is already wide enough to take into account further uncertainties by considering explicitly the $c_{i}$ 's from the IR study of Ref. [57, which indeed are rather close to those obtained in EOMS [11]. We also notice that the values from Ref. [58], obtained in a $N N$ scattering study, are very similar to those of KH [55], so that in the following we consider only the latter ones. Again the uncertainty estimated takes into account the variation in the results by employing the $c_{i}$ 's from Ref. [58]. The work [56] fixes $c_{4}$ accurately but its analysis is insensitive to $c_{3}$, precisely the $\mathcal{O}\left(p^{2}\right)$ 


\begin{tabular}{|r|l|l|l|}
\hline Analysis & $c_{1}\left[\mathrm{GeV}^{-1}\right]$ & $c_{3}\left[\mathrm{GeV}^{-1}\right]$ & $c_{4}\left[\mathrm{GeV}^{-1}\right]$ \\
\hline GW-HBChPT [55] & -1.13 & -5.51 & 3.71 \\
KH-HBChPT [55] & -0.75 & -4.77 & 3.34 \\
\hline KH [56] & $-0.81 \pm 0.12$ & $8 \pm 57$ & $3.40 \pm 0.04$ \\
\hline GW-EOMS [11] & $-1.50 \pm 0.007$ & $-6.63 \pm 0.31$ & $3.68 \pm 0.14$ \\
KH-EOMS [11] & $-1.26 \pm 0.14$ & $-6.74 \pm 0.38$ & $3.74 \pm 0.16$ \\
\hline GW-IR [57] & $-1.32 \pm 14$ & $-6.9 \pm 6$ & $3.66 \pm 0.33$ \\
KH-IR [57] & $-1.08 \pm 0.15$ & $-7.0 \pm 0.7$ & $3.72 \pm 0.32$ \\
\hline NN data [58] & $-0.76 \pm 0.7$ & $-4.78 \pm 0.10$ & $3.96 \pm 0.22$ \\
\hline GW-UChPT [11] & $-1.11 \pm 0.02$ & $-4.78 \pm 0.04$ & $3.04 \pm 0.02$ \\
KH-UChPT [11] & $-1.04 \pm 0.02$ & $-4.48 \pm 0.05$ & $3.00 \pm 0.02$ \\
\hline
\end{tabular}

Table 1: Different sets of values for the $\mathcal{O}\left(p^{2}\right) \pi N$ LECs $c_{1}, c_{3}$ and $c_{4}$.

$\pi N$ LEC on which our results mostly depend. This is why we do not show results for this set of $c_{i}$ 's either. Finally, we also give the resulting values from the fits to $\pi N$ data within Unitarized EOMS BChPT obtained in Ref. [11]. These are the fits that provide more stable values under the change of the data between $\mathrm{KH}$ and GW. These values are quite similar to those from the set KH [55]. In summary, when discussing our results we will take into account the values for the LECs $c_{i}$ obtained in Refs. [55] and [1], namely, the rows 2, 3, 5, 6, 10 and 11 in Table 1.

\subsection{Number of subtractions in the chiral expansion of $\Delta(A)$}

An interesting point to discuss is the appropriate number of subtractions for a given chiral order in the calculation of $\Delta(A)$. In other terms, we want to establish a chiral power counting for the subtraction constants involved in the calculation of the functions $D(A)$ and $N(A)$.

In the previous works in which we applied the $N / D$ method to study $N N$ interactions [42 44] our main criterion for fixing the number of subtractions was to end with a well-defined IE for $D(A)$ with $A<L$. We could also add more subtractions and fit low-energy data with more precision by having more free parameters at our disposal, a point actually used in these works too. However, by having a chiral power counting for the subtraction constants one has a connection between the number of subtraction and the chiral order for the calculation of $\Delta(A)$.

A chiral power counting for the subtraction constants can be established by studying their variation when changing the subtraction point in the low-energy region. Let us consider first the chiral order for the subtraction constants appearing in $N(A)$, denoted by $\nu_{i}$ in Eq. (6) . For definiteness let us employ a twice-subtracted DR, which reads

$$
N(A)=\nu_{1}+\nu_{2} A+\frac{A^{2}}{\pi} \int_{-\infty}^{L} d k^{2} \frac{\Delta\left(k^{2}\right) D\left(k^{2}\right)}{\left(k^{2}\right)^{2}\left(k^{2}-A\right)} .
$$

Now, let us move the subtraction point from zero to $C=\mathcal{O}\left(M_{\pi}^{2}\right)$. It is then straightforward to show that 
the previous DR can be rewritten as 6

$$
\begin{aligned}
N(A) & =\nu_{1}^{\prime}+\nu_{2}^{\prime} A+\frac{(A-C)^{2}}{\pi} \int_{-\infty}^{L} d k^{2} \frac{\Delta\left(k^{2}\right) D\left(k^{2}\right)}{\left(k^{2}-C\right)^{2}\left(k^{2}-A\right)}, \\
\nu_{1}^{\prime} & =\nu_{1}-\frac{C^{2}}{\pi} \int_{-\infty}^{L} d k^{2} \frac{\Delta\left(k^{2}\right) D\left(k^{2}\right)}{\left(k^{2}-C\right)^{2} k^{2}} \\
\nu_{2}^{\prime} & =\nu_{2}+\frac{C}{\pi} \int_{-\infty}^{L} d k^{2} \frac{\Delta\left(k^{2}\right) D\left(k^{2}\right)}{\left(k^{2}-C\right)^{2} k^{2}} \frac{2 k^{2}-C}{k^{2}} .
\end{aligned}
$$

For $C=\mathcal{O}\left(p^{2}\right), k^{2}=\mathcal{O}\left(p^{2}\right)$ because the result of the convergent integral at low-energies is dominated by the low-energy region of the integrand and $D\left(k^{2}\right)=1+\ldots=\mathcal{O}\left(p^{0}\right)$. Furthermore, since at leading order $\Delta\left(k^{2}\right)=\mathcal{O}\left(p^{0}\right)$, it follows then from Eq. (25) that $\nu_{1}=\mathcal{O}\left(p^{0}\right)$ and $\nu_{2}=\mathcal{O}\left(p^{-2}\right)$. This procedure can be easily generalized so that $\nu_{n}=\mathcal{O}\left(p^{-2(n-1)}\right)$. By increasing the chiral order in the calculation of $\Delta(A)$ up to $\mathcal{O}\left(p^{m}\right)$ the $\nu_{n}$ will receive an extra contribution starting at $\mathcal{O}\left(p^{-2(n-1)+m}\right)$, as it is also clear from Eq. (25). Now, the point is to demand that for a given $m$ the maximum value of $n$, denoted by $n_{0}$, should not be so large that $-2\left(n_{0}-1\right)+m<0$. By this condition we are requiring that the chiral dimension for a given subtraction constant with $n \leq n_{0}$ be positive or zero, since short-distance physics gives rise to contributions that do not vanish in the chiral limit 7 Then the raising in the chiral dimension of $\nu_{n}$ until the nominal one, $-2(n-1)+m \geq 0$, must come from powers of $M_{\pi},|C|^{\frac{1}{2}} \sim M_{\pi}$. 8 This power counting coincides with the standard Weinberg chiral power counting [23], that is applied to the calculation of the $N N$ potential. It is also worth noticing that $\nu_{n}$ is multiplied by $(A-C)^{n-1}$, so that the chiral order of the product is always $m$ for any $n$, which corresponds to the chiral order of the dispersive integral with the $\mathcal{O}\left(p^{m}\right)$ contribution of $\Delta(A)$.

One can proceed analogously also for the function $D(A)$. We also exemplify it by writing down a twice-subtracted DR for $D(A)$,

$$
D(A)=1+\delta_{2} A-\frac{A(A-C)}{\pi} \int_{0}^{\infty} d q^{2} \frac{\rho\left(q^{2}\right) N\left(q^{2}\right)}{q^{2}\left(q^{2}-C\right)\left(q^{2}-A\right)} .
$$

We now move the subtraction point from $C$ to $E$. Let us recall that the normalization $D(0)=1$ is fixed and this is why we do not change the position of the first subtraction taken at $A=0$. As a result of this rewriting we obtain the evolution

$$
\delta_{2} \rightarrow \delta_{2}+\frac{C-E}{\pi} \int_{0}^{\infty} d q^{2} \frac{\rho\left(q^{2}\right) N\left(q^{2}\right)}{q^{2}\left(q^{2}-E\right)\left(q^{2}-C\right)}
$$

For ascribing the chiral order to $\delta_{n}, n \geq 2$, we have, as before, that $C \sim E \sim M_{\pi}^{2}, q^{2}=\mathcal{O}\left(p^{2}\right)$. Additionally, we count $\rho\left(q^{2}\right)=\mathcal{O}\left(p^{0}\right)$ because it involves the product $m \sqrt{q^{2}}$ with $m \gg M_{\pi}$, a large number. Let us also recall at this point that along the RHC, the extent of the integral in Eq. (26), the strong effects due to the infrared enhancement of the $N N$ intermediate states [23], which is directly related

\footnotetext{
${ }^{6}$ To show this, one can rewrite the factor $A^{2} / k^{2}$ in the integral of Eq. (24) as $([A-C]+C)^{2} /\left(k^{2}-C\right)^{2} \times\left(k^{2}-C\right)^{2} /\left(k^{2}\right)^{2}$ and then isolate the term $(A-C)^{2} /\left(k^{2}-C\right)^{2}$. The rest of terms can be reabsorbed in the polynomial on the right-hand side (r.h.s.) of Eq. (24).

${ }^{7}$ As a result they are counted as $\mathcal{O}\left(p^{0}\right)$

${ }^{8}$ One could ask about the fact that the chiral dimension for the other contributions to $\Delta(A)$ of order $m^{\prime}<m$ could imply a negative $-2(n-1)+m^{\prime}$ with $n \leq n_{0}$. This already occurs e.g. in the paradigmatic example of ChPT, namely, meson-meson scattering. The point is to realize that these extra long-range physics contributions cancel explicitly with other contributions stemming from the rearrangement of the dispersive integral, which was done already with less subtractions when including only lower orders in $\Delta(A)$.
} 
with the large nucleon mass, should be resummed. We then conclude from Eq. (27) that $\delta_{2}=\mathcal{O}\left(p^{-2}\right)$ for the LO contribution of $N(A)=\mathcal{O}\left(p^{0}\right)$. This result can be generalized easily to more subtractions so that $\delta_{n}=\mathcal{O}\left(p^{-2(n-1)}\right)$. However, this chiral order increases when considering higher orders contributions to $N(A)$ stemming in turn from higher orders in the calculation of $\Delta(A)$. As just discussed above in connection with Eq. (25), these $\mathcal{O}\left(p^{m}\right)$ contributions to $\Delta(A)$ give rise to contributions of the same order in $N(A)$. Thus, once they are taken into account, one has the corresponding rise in the chiral order of $\delta_{n}$ so that now it counts as $\delta_{n}=\mathcal{O}\left(p^{-2(n-1)+m}\right)$. In this way, the chiral orders of $\nu_{n}$ and $\delta_{n}$ are the same for the same $n$ and $m$. Indeed, this is a necessary result because according with the general formalism of Sec. 2 the same number of subtractions are taken both in $D(A)$ and $N(A)$. To satisfy this requirement is also another reason for taking $\rho=\mathcal{O}\left(p^{0}\right)$ in the chiral counting. We also stress that the chiral power counting that we have established for the subtraction constants $\delta_{n}$ corresponds to two-nucleon reducible diagrams 9 while the standard Weinberg chiral power counting for nuclear interactions [23] only involves two-nucleon irreducible diagrams.

Although we have offered here the arguments for the uncoupled case the same results follow for the coupled-channel partial waves because the function $\nu_{i j}(A)$, Eq. (12), share the same chiral counting as $\rho(A)$, since the $T$-matrix is $\mathcal{O}\left(p^{0}\right) 10$ In summary, for $\Delta(A)$ calculated up to $\mathcal{O}\left(p^{m}\right)$ we have the following power counting for the subtractions constants,

$$
\nu_{n}, \delta_{n} \sim \mathcal{O}\left(p^{-2(n-1)+m}\right) .
$$

Now, by applying the requirement that $-2(n-1)+m \geq 0$ it results that in our present study at NNLO one should properly take two subtractions $(n=2)$ since $m=3$. However, on top of this criterion we first require that the resulting IE has well-defined solutions and for this to happen it is necessary to introduce more than two subtractions in some $N N$ partial waves, as discussed below. In addition, we have to satisfy the right threshold behavior for higher partial waves, which for $\ell \geq 3$ ( $J \geq 3$ for the mixing partial waves) requires to take $\ell>2$ ( $J>2$ subtractions), cf. Sec. 2.3.

\section{Uncoupled ${ }^{1} S_{0}$ wave}

In this section we study the ${ }^{1} S_{0}$ partial wave. We first take the once-subtracted DRs:

$$
\begin{aligned}
& D(A)=1-\nu_{1} A g(A, 0)+\frac{A}{\pi} \int_{-\infty}^{L} d k^{2} \frac{\Delta\left(k^{2}\right) D\left(k^{2}\right)}{k^{2}} g\left(A, k^{2}\right), \\
& N(A)=\nu_{1}+\frac{A}{\pi} \int_{-\infty}^{L} d k^{2} \frac{\Delta\left(k^{2}\right) D\left(k^{2}\right)}{k^{2}\left(k^{2}-A\right)}
\end{aligned}
$$

We have one free parameter $\nu_{1}$ that can be fixed in terms of the ${ }^{1} S_{0}$ scattering length $a_{s}$

$$
\nu_{1}=-\frac{4 \pi a_{s}}{m},
$$

with the experimental value $a_{s}=-23.76 \pm 0.01 \mathrm{fm}[32$.

The phase shifts obtained by solving the IE of Eq. (29) are shown in Fig. 1 as a function of the c.m. three-momentum, denoted by $p(p=\sqrt{A})$ in the axis of abscissas. The (red) hatched area corresponds to our results from Eqs. (29)-(31) with $\Delta(A)$ calculated up-to-and-including $\mathcal{O}\left(p^{3}\right)$ contributions and by taking into account the variation in the results from the different values employed for the NLO $\pi N$ ChPT

\footnotetext{
${ }^{9}$ As it is apparent from the factor $q^{2}-A$ in the denominator of the RHC integral in Eq. (26).

${ }^{10}$ With $\rho=\mathcal{O}\left(p^{0}\right)$ it is also true that $\operatorname{Im} t_{i j}=\mathcal{O}\left(p^{0}\right)$ because of unitarity for $A \geq 0, \operatorname{Im} t_{i j}=\rho \sum_{k} t_{i k} t_{j k}^{*}$.
} 


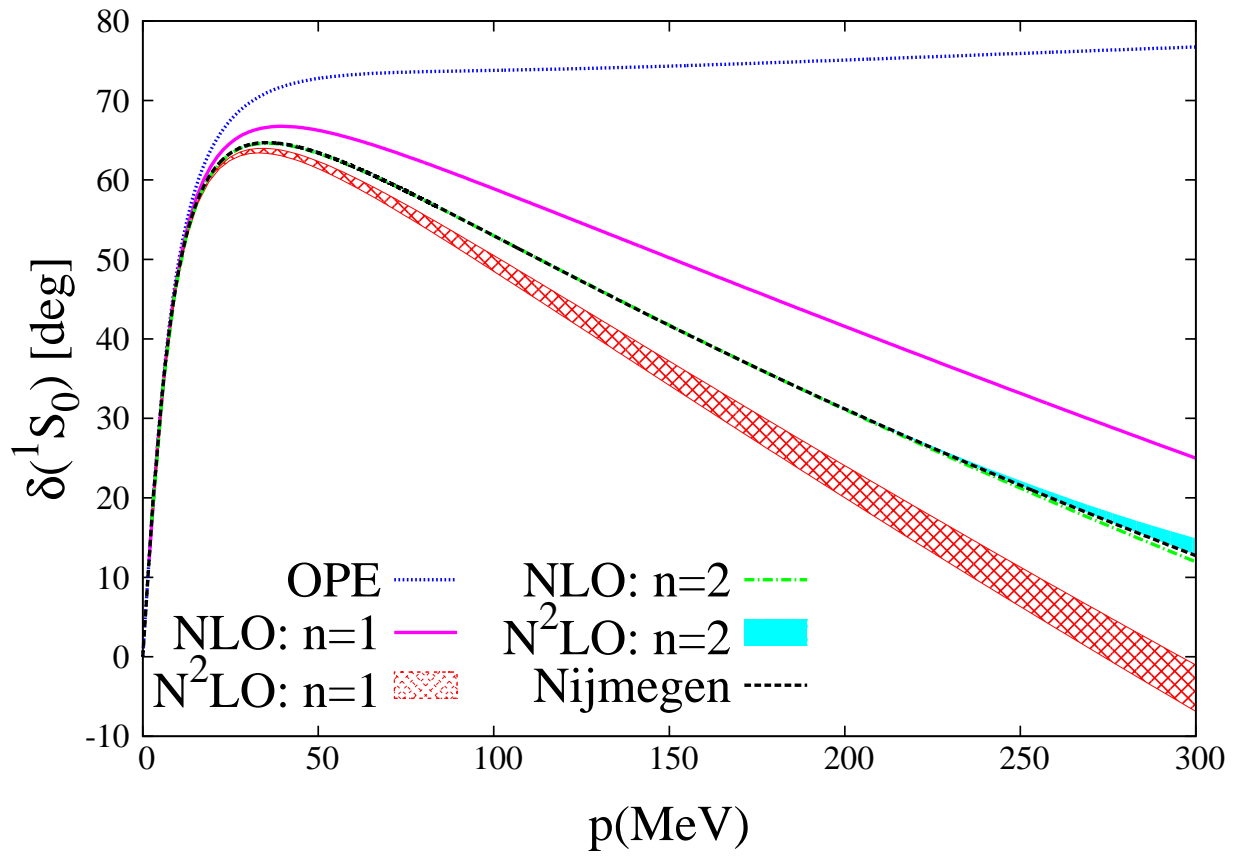

Figure 1: (Color online.) Phase shifts of the ${ }^{1} S_{0} N N$ partial wave where the number of subtractions taken is indicated by the value of $n$ given in the legend of each type of line. The once-subtracted DR results are shown by the (red) hatched areas at NNLO, the (magenta) solid lines at NLO [44] and the (blue) dotted lines at LO (OPE) [42]. The twice-subtracted DR results correspond to the (cyan) band at NNLO and the (green) dash-dotted line at NLO [44]. The Nijmegen PWA phase shifts are shown by the (black) dashed lines.

counterterms in Table 1. Our present results are compared with the neutron-proton $(n p){ }^{1} S_{0}$ phase shifts of the Nijmegen PWA [45] (black dashed line), the OPE results of Ref. [42] (blue dotted line) and the NLO results of Ref. [44] (magenta solid line). As we see, the Nijmegen PWA phase shifts are better reproduced at lower energies at NNLO than at smaller orders, though one also observes an excess of repulsion at this order.

Next, we work out the effective range expansion (ERE) parameters for the ${ }^{1} S_{0}$. By taking into account the relation in our normalization

$$
\frac{4 \pi}{m} \frac{D}{N}=-\frac{1}{a_{s}}+\frac{1}{2} r_{s} A+\sum_{i=1}^{10} v_{i} A^{i}-i \sqrt{A}+\mathcal{O}\left(A^{11}\right),
$$

with $r_{s}$ the ${ }^{1} S_{0}$ effective range and the shape parameters $v_{i}, i=2, \ldots, 10$. We designate by $I_{m}, m=$ $1,2, \ldots$, the integral along the LHC,

$$
\begin{aligned}
I_{2 n} & =\int_{-\infty}^{L} d k^{2} \frac{\Delta\left(k^{2}\right) D\left(k^{2}\right)}{\left(k^{2}\right)^{n}}, \\
I_{2 n+1} & =\int_{-\infty}^{L} d k^{2} \frac{\Delta\left(k^{2}\right) D\left(k^{2}\right)}{\left(k^{2}\right)^{n} \sqrt{-k^{2}}} .
\end{aligned}
$$




\begin{tabular}{|l|l|l|l|l|l|l|}
\hline & $r_{s}$ & $v_{2}$ & $v_{3}$ & $v_{4}$ & $v_{5}$ & $v_{6}$ \\
\hline NLO & 2.32 & -1.08 & 6.3 & -36.2 & 225 & -1463 \\
\hline NNLO-I & $2.92(6)$ & $-0.32(8)$ & $4.9(1)$ & $-27.7(8)$ & $177(4)$ & $-1167(30)$ \\
\hline NNLO-II & $2.699(4)$ & $-0.657(3)$ & $5.20(2)$ & $-30.39(9)$ & $191.9(6)$ & $-1263(3)$ \\
\hline Ref. [32] & 2.68 & -0.61 & 5.1 & -30.0 & & \\
\hline Ref. [48] & $2.62 \sim 2.67$ & $-0.52 \sim-0.48$ & $4.0 \sim 4.2$ & $-20.5 \sim-19.9$ & & \\
\hline Ref. [45] & 2.68 & -0.48 & 4.0 & -20.0 & & \\
\hline
\end{tabular}

Table 2: Values for effective range $r_{s}[\mathrm{fm}]$ and the shape parameters $v_{i}, i=2, \ldots, 6$ in units of fm ${ }^{2 i-1}$ for our present results at NNLO with once-subtracted DRs [Eq. (29)] (NNLO-I in the third row) and with twice-subtracted DRs [Eq. (38)] (NNLO-II in the fourth row). The second row shows the results at NLO with once-subtracted DRs [Eq. (29)]. We also give the values obtained by using the NNLO $N N$ potential in Refs. 32 and [48] (fifth and sixth rows, respectively). The values corresponding to the Nijmegen PWA [45], as obtained in Refs. [32, 48, are given in the last row.

From Eqs. (29), (30) and (32) we derive the following expressions for $r_{s}$ and the shape parameters in the ERE up to $i=4$

$$
\begin{aligned}
r_{s} & =-\frac{m\left(a_{s} I_{3}+I_{4}\right)}{2 \pi^{2} a_{s}^{2}} \\
v_{2} & =-\frac{m\left(I_{4} m\left(a_{s} I_{3}+I_{4}\right)+4 \pi^{2} a_{s}\left(a_{s} I_{5}+I_{6}\right)\right)}{16 \pi^{4} a_{s}^{3}}, \\
v_{3} & =-\frac{m\left[16 \pi^{4} a_{s}^{2}\left(a_{s} I_{7}+I_{8}\right)+I_{4}^{2} m^{2}\left(a_{s} I_{3}+I_{4}\right)+4 \pi^{2} a_{s} m\left(a_{s} I_{3} I_{6}+a_{s} I_{4} I_{5}+2 I_{4} I_{6}\right)\right]}{64 \pi^{6} a_{s}^{4}} \\
v_{4} & =-\frac{m}{256 \pi^{8} a_{s}^{5}}\left[64 \pi^{6} a_{s}^{3}\left(a_{s} I_{9}+I_{10}\right)+16 \pi^{4} a_{s}^{2} m\left(a_{s}\left(I_{3} I_{8}+I_{4} I_{7}+I_{5} I_{6}\right)+2 I_{4} I_{8}+I_{6}^{2}\right)+I_{4}^{3} m^{3}\left(a_{s} I_{3}+I_{4}\right)\right. \\
& \left.+4 \pi^{2} a_{s} I_{4} m^{2}\left(2 a_{s} I_{3} I_{6}+a_{s} I_{4} I_{5}+3 I_{4} I_{6}\right)\right] .
\end{aligned}
$$

For higher order shape parameters is more efficient to use the numerical method developed in Appendix A, to which we refer.

The resulting values for $r_{s}$ and the shape parameters $v_{i}, i=1, \ldots, 6$, are given in Table 2 and for $v_{i}$, $i=7, \ldots, 10$ are shown in Table 3 in the second and third rows for NLO and NNLO, respectively. The latter are indicated by NNLO-I. These results are compared with the results from the calculation based on the NNLO $N N$ potential of Refs. [48] and [32], and with the Nijmegen PWA values. Our results for $v_{3}$ and $v_{4}$ are very similar to those obtained in Ref. [32]. The difference between [32] and [48] stems from the fact that in the latter reference a different method to regularize pion exchanges was introduced, the so-called spectral function regularization, instead of the dimensional regularization used in Ref. [32]. We also observe a clear improvement in the reproduction of the ERE parameters from NLO to NNLO. At NLO the errors in Tables 2 and 3 reflect the numerical uncertainty in the calculation of higher order derivatives. At NNLO in addition they take into account the spread in the results from the different sets of $c_{i}$ 's used.

From Eq. (35) we can also derive a power series expansion of the ERE parameters as a function of $a_{s}$, as it was done previously for $r_{s}$ in Ref. [4] at NLO. We refer to that reference for further details. The important point is that $D(A)$ satisfies the linear IE of Eq. (29) with an inhomogeneous term that is a polynomial of first degree in $a_{s}$. As a result, $D(A)=D_{0}(A)+a_{s} D_{1}(A)$, with $D_{0}(A)$ and $D_{1}(A)$ 


\begin{tabular}{|l|l|l|l|l|}
\hline & $v_{7} \times 10^{-1}$ & $v_{8} \times 10^{-2}$ & $v_{9} \times 10^{-3}$ & $v_{10} \times 10^{-4}$ \\
\hline NLO & 985 & -681 & 480 & $-344(3)$ \\
\hline NNLO-I & $795(18)$ & $-554(12)$ & $393(8)$ & $-284(6)$ \\
\hline NNLO-II & $857.1(1.9)$ & $-595.7(1.3)$ & $421.7(9)$ & $-304(3)$ \\
\hline
\end{tabular}

Table 3: Values for the shape parameter $v_{i}, i=7, \ldots, 10$ in units of $\mathrm{fm}^{2 i-1}$. For the meanings of the rows see Table 2 ,

independent of $a_{s}$. This also implies that the different $I_{n}$ can be expressed as $I_{n}^{(0)}+a_{s} I_{n}^{(1)}$ with $I_{n}^{(0)}$ and $I_{n}^{(1)}$ independent of $a_{s}$. In this way, the ERE parameters satisfies the following expansions

$$
\begin{aligned}
& r_{s}=\alpha_{0}+\frac{\alpha_{-1}}{a_{s}}+\frac{\alpha_{-2}}{a_{s}^{2}}, \\
& v_{n}=\sum_{m=-n-1}^{0} \frac{v_{n}^{(m)}}{a_{s}^{m}},
\end{aligned}
$$

with the coefficients $\alpha_{i}$ and $v_{n}^{(i)}$ independent of $a_{s}$. The relation between $r_{s}$ and $a_{s}$ was first realized in Ref. [34] in the context of $N N$ scattering 11 The explicit expressions of $\alpha_{i}(i=-2,-1,0)$ in terms of $D_{0}(A)$ and $D_{1}(A)$ were given in Ref. [44]. Its values at NNLO are

$$
\begin{aligned}
\alpha_{0} & =2.61 \sim 2.73 \mathrm{fm}, \\
\alpha_{-1} & =-5.93 \sim-5.65 \mathrm{fm}^{2}, \\
\alpha_{-2} & =5.92 \sim 6.12 \mathrm{fm}^{3} .
\end{aligned}
$$

The expressions for the coefficients $v_{n}^{(m)}$ in Eq. (36) can also be worked straightforwardly in terms of $D_{0}(A)$ and $D_{1}(A)$ by the interested reader. For conciseness we do not reproduce them here. The results in Eq. (37) are perfectly compatible with those obtained in the first entry of Ref. [34], $\alpha_{0}=2.59 \sim 2.67 \mathrm{fm}$, $\alpha_{-1}=-5.85 \sim-5.64 \mathrm{fm}^{2}$ and $\alpha_{-2}=5.95 \sim 6.09 \mathrm{fm}^{3}$. This reference employs the chiral $N N$ potential in a Lippmann-Schwinger equation that is renormalized with boundary conditions and imposing the hypothesis of orthogonality of the wave functions determined with different energy 12 In our case, however, the expansions in Eq. (36) are consequences of basic principles of a $N N$ partial wave like unitarity, analyticity and chiral symmetry. The resulting phase shifts in Fig. 11 from Eq. (29), and shown by the (red) hatched area, are also coincident with those obtained by Ref. [34]. They are also rather similar to those obtained when employing only one contact term in the third entry of Ref. [37], which studies the independence of its results as a function of the cutoff used to solve the Lippmann-Schwinger equation. Nevertheless, in this case the NNLO chiral potential is calculated by truncating its spectral representation [48, while Ref. 34] uses the dimensional regularized result (which requires to take to infinity the cutoff(s) used in Ref. [37].)

\footnotetext{
${ }^{11}$ The correlation between the effective range and the scattering length in Eq. (36) was derived earlier in atomic physics for Van der Waals potentials [60, and throughly confronted with data 61.

${ }^{12}$ Since the potentials involved are singular this orthogonality condition is imposed in the formalism of Ref. 34.
} 
Next, we consider the twice-subtracted DRs:

$$
\begin{aligned}
D(A) & =1+\delta_{2} A-\nu_{1} \frac{A\left(A+M_{\pi}^{2}\right)}{\pi} \int_{0}^{\infty} d q^{2} \frac{\rho\left(q^{2}\right)}{\left(q^{2}-A\right)\left(q^{2}+M_{\pi}^{2}\right) q^{2}}-\nu_{2} A\left(A+M_{\pi}^{2}\right) g\left(A,-M_{\pi}^{2}\right) \\
& +\frac{A\left(A+M_{\pi}^{2}\right)}{\pi^{2}} \int_{-\infty}^{L} d k^{2} \frac{\Delta\left(k^{2}\right) D\left(k^{2}\right)}{\left(k^{2}\right)^{2}} \int_{0}^{\infty} d q^{2} \frac{\rho\left(q^{2}\right) q^{2}}{\left(q^{2}-A\right)\left(q^{2}+M_{\pi}^{2}\right)\left(q^{2}-k^{2}\right)}, \\
N(A) & =\nu_{1}+\nu_{2} A+\frac{A^{2}}{\pi} \int_{-\infty}^{L} d k^{2} \frac{\Delta\left(k^{2}\right) D\left(k^{2}\right)}{\left(k^{2}-A\right)\left(k^{2}\right)^{2}},
\end{aligned}
$$

where the two subtractions in the function $N(A)$ and one for $D(A)$ are taken at $C=0$, while the other subtraction in $D(A)$ is placed at $C=-M_{\pi}^{2}$. Taking into account Eq. (9) it is straightforward to rewrite

$$
\frac{1}{\pi} \int_{0}^{\infty} d q^{2} \frac{\rho\left(q^{2}\right) q^{2}}{\left(q^{2}-A\right)\left(q^{2}-k^{2}\right)\left(q^{2}-C\right)}=\frac{C g(A, C)-k^{2} g\left(A, k^{2}\right)}{C-k^{2}}
$$

The subtraction constant $\nu_{1}$ is given by Eq. (31), while $\nu_{2}$ and $\delta_{2}$ are directly fitted to the $n p$ Nijmegen PWA phase shifts 13 The best fit occurs for

$$
\begin{aligned}
& \nu_{2}=-23(1) M_{\pi}^{-4} \\
& \delta_{2}=-8.0(3) M_{\pi}^{-2},
\end{aligned}
$$

where the intervals of values stem from the uncertainty due to the different values of $c_{i}$ 's taken. The reproduction of data is very good, as shown by the (cyan) filled area in Fig. 1 which lies on top of the Nijmegen PWA $n p$ phase shifts. In the same figure we show by the (green) dash-dotted line the twicesubtracted DR result at NLO, which reproduces the Nijmegen data equally well as obtained at NNLO, with the fitted values $\nu_{2}=-11.9 M_{\pi}^{-4}$ and $\delta_{2}=-4.6 M_{\pi}^{-2}$. The resulting ERE shape parameters for the fit in Eq. (41) are shown in the fourth rows of Tables 2 and 3 , where we observe a remarkable good agreement with Ref. [32]. We predict $r_{s}=2.70 \mathrm{fm}$ which is compatible with its experimental value $r_{s}=2.75 \pm 0.05 \mathrm{fm}$ [32]. A similar good reproduction of the ${ }^{1} S_{0}$ phase shifts is also achieved by Ref. [37] in terms of two contact terms, although in this case there is a strong sensitivity on the cutoff employed to regularize the Lippmann-Schwinger equation near those values that give rise to poles in the domain of validity of the effective field theory.

The value of $\nu_{2}$ in Eq. (41) is rather large, of similar size in absolute value to $\nu_{1} \simeq 31 M_{\pi}^{-2}$, Eq. (31). A linear correlation between $\nu_{2}$ and $\delta_{2}$ can be observed in a $\chi^{2}$ contour plot, along which there is an absolute minimum corresponding to the parameters given in Eq. (41). The subtraction constant $\nu_{2}$ that results from the once-subtracted DR Eq. (30), and that we denote by $\nu_{2}^{\text {pred }}$, is given by the expression

$$
\nu_{2}^{\text {pred }}=\frac{1}{\pi} \int_{-\infty}^{L} d k^{2} \frac{\Delta\left(k^{2}\right) D\left(k^{2}\right)}{\left(k^{2}\right)^{2}},
$$

with the numerical value $\nu_{2}^{\text {pred }} \simeq-6.0,-6.4$ and $-7.5 \pm 0.2 M_{\pi}^{-4}$ when $\Delta(A)$ is calculated up to $\mathcal{O}\left(p^{0}\right)$, $\mathcal{O}\left(p^{2}\right)$ and $\mathcal{O}\left(p^{3}\right)$, respectively. The difference between the predicted and fitted values for $\nu_{2}$ at NLO is denoted by $\delta \nu_{2}^{(0)}$. The superscript takes into account the chiral order for $\nu_{2}, \mathcal{O}\left(p^{-2+m}\right)$ according to the new contribution to $\Delta(A)$ of $\mathcal{O}\left(p^{m}\right)$, Eq. (28) . The value obtained is $\delta \nu_{2}^{(0)} \simeq-5.5 M_{\pi}^{-4}$. At NNLO in order to calculate $\delta \nu_{2}^{(1)}$ one has to subtract $\delta \nu_{2}^{(0)}$ to the difference between the fitted value in Eq. (41) and the predicted one from Eq. (42). Then, one has $\delta \nu_{2}^{(1)} \simeq-15+5.5=-9.5 M_{\pi}^{-4}$. This implies that

\footnotetext{
${ }^{13}$ Since Ref. [45] does not provide errors we always perform a least square fit, without weighting.
} 
in order to overcome the excess of repulsion at NNLO one needs to incorporate a significant contribution from short-distance physics to give account of "missing physics", beyond the pure long-range physic: 14 that stems from the once-subtracted DR case and that is not able to provide an accurate reproduction of data as shown in Fig. 1 by the (red) hatched areas. The large value for $\delta \nu_{2}^{(1)}$ is mainly due to the $\mathcal{O}\left(p^{2}\right)$ $\pi N$ counterterms $c_{i}$ 's, which in turn are dominated by the $\Delta(1232)$ resonance contribution 62,63 . This can be easily seen by performing a fit to data in which we set $c_{i}=0$ for all of them. A good reproduction of the Nijmegen PWA phase shifts results but now $\delta \nu_{2}^{(1)} \simeq-1.5 M_{\pi}^{-4}$, which is much smaller than $\delta \nu_{2}^{(0)}$, with a ratio $\delta \nu_{2}^{(1)} / \delta \nu_{2}^{(0)} \sim 30 \% \sim \mathcal{O}(p)$. This indicates that once the large contributions that stem from the $c_{i}$ coefficients are discounted a quite natural (baryon) chiral expansion emerges.

Regarding the absolute value of $\delta \nu_{2}^{(0)}$ one should expect on dimensional grounds that

$$
\left|\delta \nu_{2}^{(0)}\right| \sim \frac{4 \pi\left|a_{s}\right|}{m \Lambda^{2}}
$$

with $\Lambda$ the expansion scale. The factor $4 \pi / m$ is due to our normalization, cf. Eq. (32). There should be also another contribution to $\delta \nu_{2}^{(0)}$ not proportional to $a_{s}$, but since the scattering length is so large the contribution shown in Eq. (43) is expected to be the most important. For $\Lambda \simeq 350 \mathrm{MeV}$, one would have $\left|\delta \nu_{2}^{(0)}\right| \sim 5 M_{\pi}^{-4}$, which is very similar indeed to the reported value above. This value of $\Lambda$ is also consistent with the ratio $\delta \nu_{2}^{(1)} / \delta \nu_{2}^{(0)} \sim 1 / 3$ given above as $M_{\pi} / \Lambda \sim 1 / 3$.

Let us consider now the relevance of the different contributions to $\Delta(A)$ by evaluating the double integral in Eq. (38), namely,

$$
\frac{A\left(A+M_{\pi}^{2}\right)}{\pi^{2}} \int_{-\infty}^{L} d k^{2} \frac{\Delta\left(k^{2}\right) D\left(k^{2}\right)}{\left(k^{2}\right)^{2}} \int_{0}^{\infty} d q^{2} \frac{\rho\left(q^{2}\right) q^{2}}{\left(q^{2}-A\right)\left(q^{2}+M_{\pi}^{2}\right)\left(q^{2}-k^{2}\right)},
$$

with the full result for $D(A)$ but with $\Delta(A)$ in the integrand of Eq. (44) evaluated partially with some contributions or all of them. The result of this exercise is given in the left panel of Fig. 2 for the $c_{i}$ coefficients of Ref. [55], collected in the first row of Table 1. In turn, we show directly $\Delta(A)$ along the LHC in the right panel of Fig. 2. The (black) dash-dotted lines correspond to OPE, the (blue) dotted lines take into account the full $\mathcal{O}\left(p^{2}\right)$ TPE, including both two-nucleon reducible and irreducible TPE, and the (cyan) double-dotted lines contain the $\mathcal{O}\left(p^{3}\right)$ two-nucleon irreducible TPE. In the right panel we show by the (cyan) filled area the variation in the $\mathcal{O}\left(p^{3}\right)$ irreducible TPE contribution by varying between the different sets of $c_{i}$ 's from Refs. [48] and [11, as discussed above. This band indicates a large source of uncertainty in $\Delta(A)$. In the left panel the (red) solid line results by keeping all the contributions to $\Delta(A)$, and one can quantify from this panel the fact that the $\mathcal{O}\left(p^{3}\right)$ irreducible TPE is the largest subleading contribution. At $\sqrt{A}=100 \mathrm{MeV}$ it is around $28 \%$ of the OPE contribution, and it raises with energy so that at $\sqrt{A}=200 \mathrm{MeV}$ it is $44 \%$ and at $300 \mathrm{MeV}$ it becomes $66 \%$. The increase in energy of the relative size of the subleading TPE contribution should be expected because at low energies the suppression mechanism due to the earlier onset of the OPE source of $\Delta(A)$ along the LHC at $L$ is more efficient. In addition, it is well-known that the $\Delta(1232)$ plays a prominent role in $\pi N$ scattering because its proximity to the $\pi N$ threshold and its strong coupling to this channel. This manifests in the large size of the LECs $c_{3}$ and $c_{4}$ in Table 1 due to the $\Delta(1232)$ contribution to them, evaluated in Refs. 62, 63]. The large impact of the $\Delta(1232)$ is the well-known reason for the large size of subleading TPE, but once its leading effects are taken into account at $\mathcal{O}\left(p^{3}\right)$ the chiral expansion stabilizes [31, 48], as we have also concluded in the discussion following Eq. (42). In the following, we skip the discussion on the relative importance of the different contributions to $\Delta(A)$ for those $N N$ partial waves with a similar situation to the one discussed concerning the ${ }^{1} S_{0}$.

\footnotetext{
${ }^{14}$ We mean here the physics driven by the multi-pion exchanges giving rise to the LHC and to $\Delta(A)$.
} 

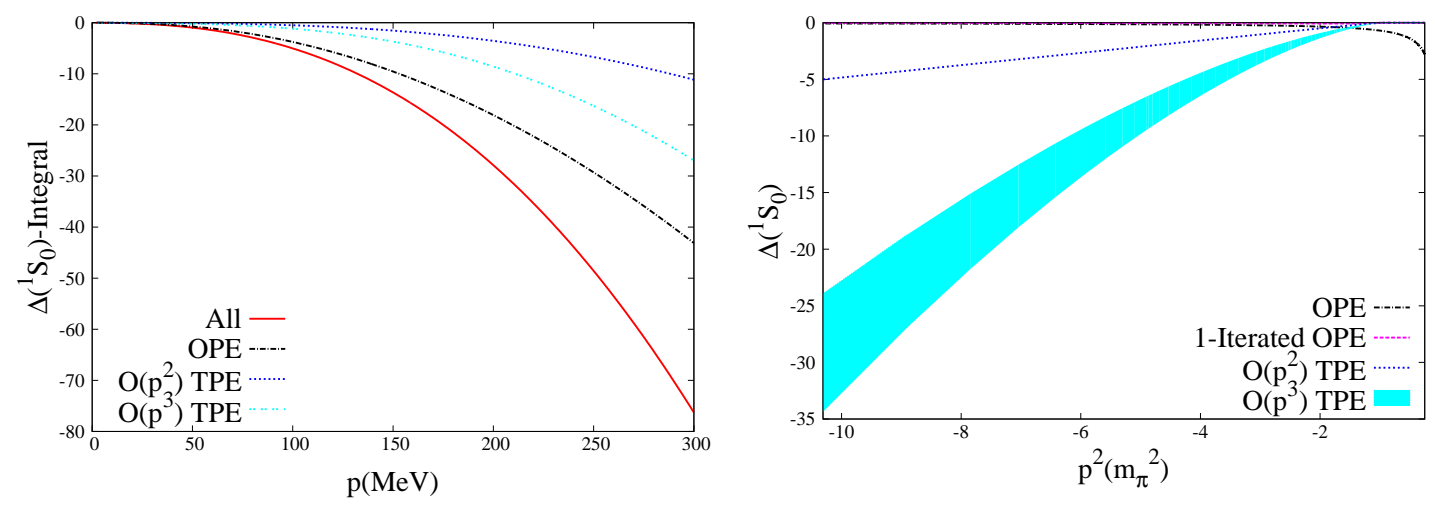

Figure 2: (Color online.) Left panel: different contributions to the integral in Eq. (44) for the ${ }^{1} S_{0}$. Right panel: contributions to $\Delta(A)$. These contributions comprise OPE (black dash-dotted line), leading TPE (blue dotted line) and the subleading TPE contribution, shown by the (cyan) double-dotted line in the left panel and by the (cyan) filled area in the right one. The total result, only shown for the left panel, is the (red) solid line.

\section{Uncoupled $P$ waves}

In this section we discuss the application of the method to the uncoupled $P$ waves. At NNLO one has for these waves that

$$
\lambda_{P}=\lim _{A \rightarrow-\infty} \frac{\Delta(A)}{(-A)^{(3 / 2)}}>0
$$

so that, according to the results of Ref. [44], its Proposition 4, a once-subtracted DR for $D(A)$, Eq. (6), does not converge and more subtractions should be taken. Then, we directly discuss the twice- and three-time subtracted DRs.

The twice-subtracted DRs are given by:

$$
\begin{aligned}
& D(A)=1+\delta_{2} A-\nu_{2} A^{2} g(A, 0)+\frac{A^{2}}{\pi} \int_{-\infty}^{L} d k^{2} \frac{\Delta\left(k^{2}\right) D\left(k^{2}\right)}{\left(k^{2}\right)^{2}} g\left(A, k^{2}\right), \\
& N(A)=\nu_{2} A+\frac{A^{2}}{\pi} \int_{-\infty}^{L} d k^{2} \frac{\Delta\left(k^{2}\right) D\left(k^{2}\right)}{\left(k^{2}-A\right)\left(k^{2}\right)^{2}}
\end{aligned}
$$

with all the subtractions in Eq. (6) taken at $C=0$. The three-time subtracted DRs are:

$$
\begin{aligned}
D(A) & =1+\delta_{2} A+\delta_{3} A\left(A+M_{\pi}^{2}\right)+\left(\nu_{2}-\nu_{3} M_{\pi}^{2}\right) A\left(A+M_{\pi}^{2}\right)^{2} \frac{\partial g\left(A,-M_{\pi}^{2}\right)}{\partial M_{\pi}^{2}}-\nu_{3} A\left(A+M_{\pi}^{2}\right)^{2} g\left(A,-M_{\pi}^{2}\right) \\
& +\frac{A\left(A+M_{\pi}^{2}\right)^{2}}{\pi} \int_{-\infty}^{L} d k^{2} \frac{\Delta\left(k^{2}\right) D\left(k^{2}\right)}{\left(k^{2}\right)^{3}} g\left(A, k^{2},-M_{\pi}^{2} ; 2\right) \\
N(A) & =\nu_{2} A+\nu_{3} A^{2}+\frac{A^{3}}{\pi} \int_{-\infty}^{L} d k^{2} \frac{\Delta\left(k^{2}\right) D\left(k^{2}\right)}{\left(k^{2}-A\right)\left(k^{2}\right)^{3}}
\end{aligned}
$$

Here all the subtractions in $N(A)$ and one in $D(A)$ are taken at $C=0$, while the other two subtractions in $D(A)$ are taken at $C=-M_{\pi}^{2}$. This is done in order to avoid handling an infrared diverging integral 


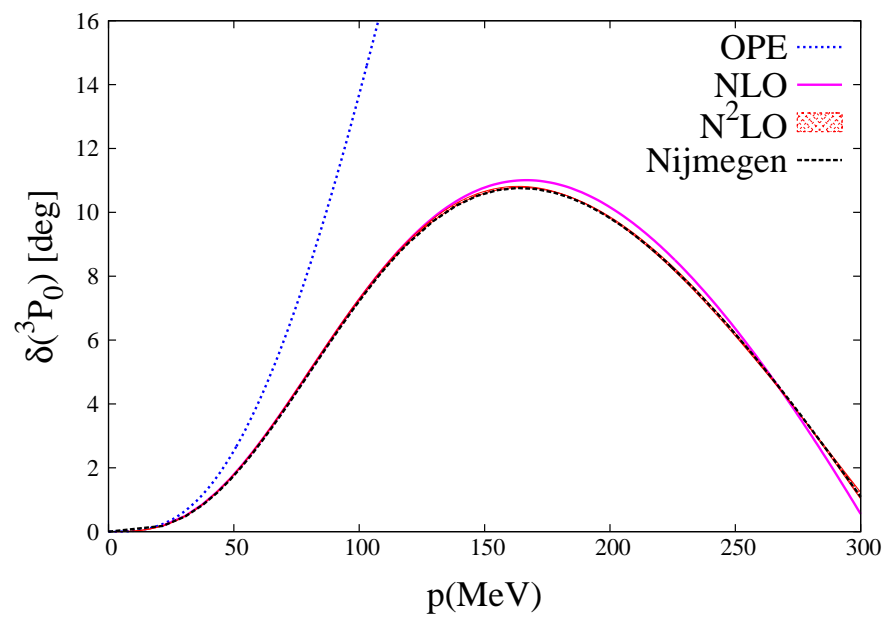

Figure 3: $\quad$ Phase shifts of the ${ }^{3} P_{0} N N$ partial wave. The three-time subtracted DR results at NNLO are shown by the (red) hatched area and the twice-subtracted DR results at NLO [44] are given by the (magenta) solid line. The (blue) dotted line corresponds to the OPE results [42] and the Nijmegen PWA phase shifts are shown by the (black) dashed lines.

along the RHC multiplying $\nu_{2}$ that would result if all the subtractions were taken at $C=0$. The function $g\left(A, k^{2}, C ; m\right)$ appearing in Eq. (47) is defined as

$$
g\left(A, k^{2}, C ; m\right)=\int_{0}^{\infty} d q^{2} \frac{\rho\left(q^{2}\right)\left(q^{2}\right)^{m}}{\left(q^{2}-A\right)\left(q^{2}-k^{2}\right)\left(q^{2}-C\right)^{m}} .
$$

In all the cases the subtraction constant $\nu_{2}$ is fixed in terms of the scattering volume, $a_{V}$,

$$
\nu_{2}=4 \pi a_{V} / m \text {. }
$$

For $a_{V}$ we take the values $0.890,-0.543$ and $-0.939 M_{\pi}^{-3}$ for the partial waves ${ }^{3} P_{0},{ }^{3} P_{1}$ and ${ }^{1} P_{1}$, in order, as deduced from Ref. [45].

\section{$5.1{ }^{3} P_{0}$ wave}

For the ${ }^{3} P_{0}$ wave the twice-subtracted DRs at NNLO, Eq. (46), do not provide stable results under the increase in absolute value of the lower limit of integration along the LHC. However, the threetime subtracted DRs, Eq. (47), are convergent and provide meaningful results. Notice that, as stated in Sec. 3.2, on top of the number of subtractions required by the chiral counting, two at NNLO, we impose the requirement of having well-defined IEs providing stable solutions. Regarding the subtractions constants $\nu_{3}, \delta_{2}$ and $\delta_{3}$ in Eq. (47), we can fix $\nu_{3}=0$ because it plays a negligible role in the fits and, if released, the fit remains stable. The fitted values for $\delta_{2}$ and $\delta_{3}$ are

$$
\begin{aligned}
& \delta_{2}=2.82(5) M_{\pi}^{-2} \\
& \delta_{3}=0.18(6) M_{\pi}^{-4},
\end{aligned}
$$

where the intervals of values take into account the dispersion in the results that stems from the different sets of $c_{i}$ 's in Table 1. The phase shifts calculated, shown by the (red) hatched area in Fig. 3, reproduce 


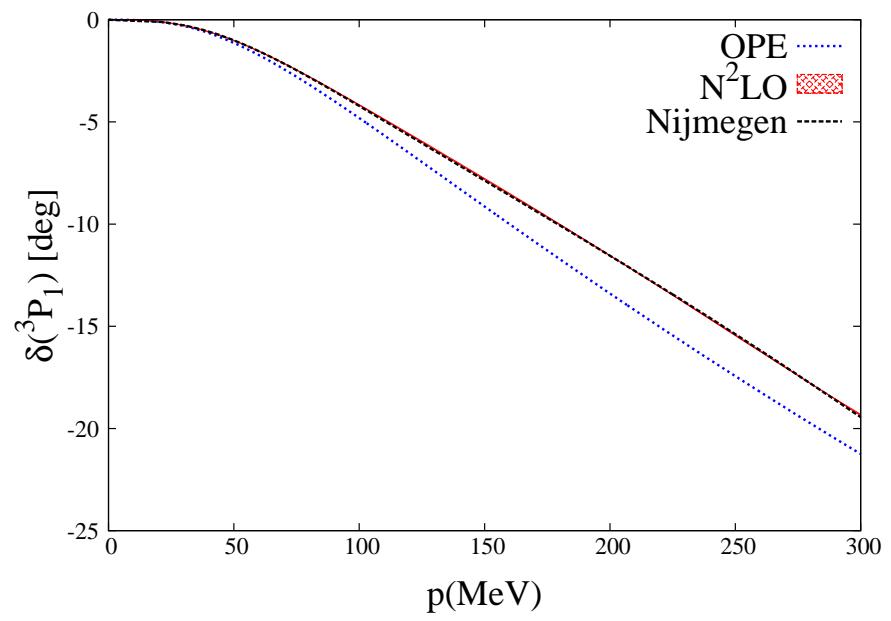

Figure 4: $\quad$ Phase shifts of the ${ }^{3} P_{1} N N$ partial wave. The three-time subtracted DR results at NNLO are shown by the (red) hatched area. The (blue) dotted line corresponds to the OPE results [42] and the Nijmegen PWA phase shifts are shown by the (black) dashed lines.

exactly the Nijmegen PWA phase shifts [45], given by the (black) dashed line. Indeed, the two lines overlap each other. The results with different sets of values for the $c_{i}$ counterterms cannot be distinguished either between each other. The (magenta) solid line shows the results with twice-subtracted DRs at NLO [44, which are already almost on top of the data, and the OPE results [42] are shown by the (blue) dotted line. We have also checked that a tree-time-subtracted DR at LO and NLO provide already a prefect reproduction of data as well. Then, the wave ${ }^{3} P_{0}$ studied at $\mathcal{O}\left(p^{3}\right)$ is not a good partial wave to learn above chiral dynamics, because independently of order up to which $\Delta(A)$ is calculated the reproduction of data is excellent when three-subtractions are taken.

\section{$5.2{ }^{3} P_{1}$ wave}

For this partial wave the situation is similar to that discussed for the ${ }^{3} P_{0}$. The twice-subtracted DRs, Eq. (46), do not provide stable results and we have to consider then the three-time subtracted DRs, Eq. (47). The free parameters are $\delta_{2}$ and $\delta_{3}$, with $\nu_{3}$ fixed to 0 (the fit is stable if this subtraction constant is released). The fitted values are

$$
\begin{aligned}
& \delta_{2}=2.7(1) M_{\pi}^{-2}, \\
& \delta_{3}=0.47(3) M_{\pi}^{-4} .
\end{aligned}
$$

The resulting phase shifts are shown in Fig. 4 by the (red) hatched area and reproduce perfectly the Nijmegen PWA phase shifts (shown by the black dashed line), independently of the set of values for the $c_{i}$ 's chosen from Refs. [11,55] in Table 1. At NLO [44] it is also necessary to take three-subtracted DRs in order to obtain stable results and the reproduction of data is equally perfect. This is why we have not included the NLO results in Fig. 4. Similarly to the ${ }^{3} P_{0}$ case, we cannot discern the impact of chiral dynamics at $\mathcal{O}\left(p^{3}\right)$ once three-time subtracted DRs are considered. 


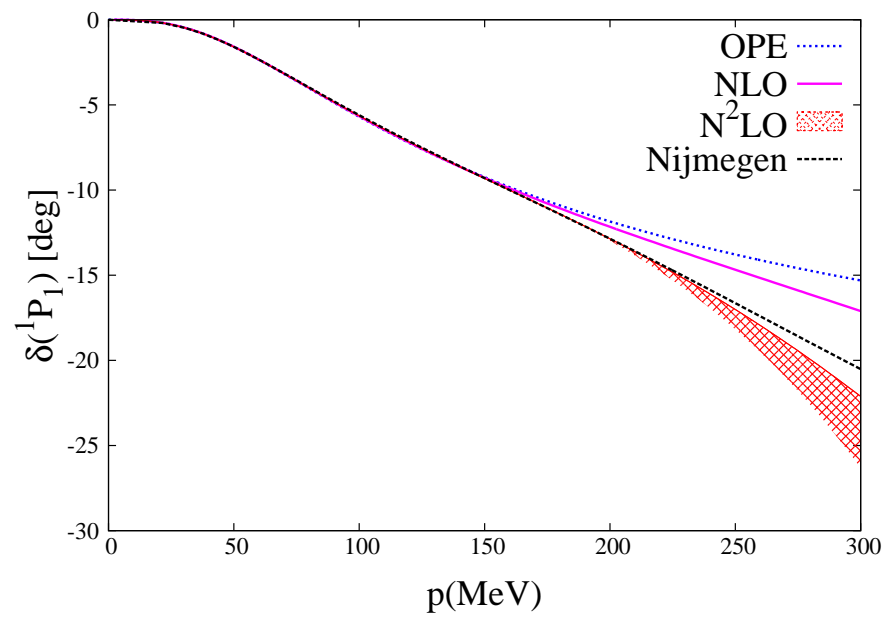

Figure 5: $\quad$ Phase shifts of the ${ }^{1} P_{1} N N$ partial wave. The twice subtracted DR results at NNLO are shown by the (red) hatched area, while at NLO [44] correspond to the (magenta) solid line. The (blue) dotted line corresponds to the OPE results [42] and the Nijmegen PWA phase shifts are shown by the (black) dashed lines.

\section{$5.3{ }^{1} P_{1}$ wave}

For this partial wave the twice-subtracted DR results from Eq. (46) are quite stable at low energies. The free parameters are now $\nu_{2}$ and $\delta_{2}$. The resulting fitted value for $\delta_{2}$ to the Nijmegen PWA phase shifts is

$$
\delta_{2}=0.4(1) M_{\pi}^{-2},
$$

with the variation in the value due to the set of $c_{i}$ 's taken $\left[\nu_{2}\right.$ is given by Eq. (49)]. We show by the (red) hatched area in Fig. 5 our results by employing the different $c_{i}$ sets of values. For this case the curves obtained with the $c_{i}$ from [11, by reproducing the $\pi N$ phase shifts with Lorentz covariant EOMS BChPT, are the closest to data and determine the upper limit of the hatched area in Fig. 5. The improvement in the reproduction of data for the ${ }^{1} P_{1}$ partial wave by the twice-subtracted DRs at NNLO compared with the results obtained at NLO with the same number of subtractions (hatched area versus (magenta) solid line) is a notorious effect from $\pi N$ physics. One should notice that for the ${ }^{1} P_{1}$ wave the dispersive integral on the r.h.s. of Eq. (46) for the function $D(A)$ is clearly dominated by the OPE contribution This is the reason why for the ${ }^{1} P_{1}$ one does not need to take three subtractions but two are enough. Although, as much as for the other partial waves discussed until now, the $\mathcal{O}\left(p^{3}\right)$ two-nucleon irreducible TPE is the dominant contribution between the subleading effects to $\Delta(A)$.

\section{Uncoupled $D$ waves}

Here, we discuss the $D$ waves. In order to preserve the right threshold behavior we employ the twicesubtracted DRs of Eqs. (17) and (18) with $\ell=2$. For the uncoupled $D$ waves one has that

$$
\lambda_{D}=\lim _{A \rightarrow-\infty} \frac{\Delta(A)}{(-A)^{3 / 2}}<0
$$

and for this sign we do not have numerical problems in the solution of the resulting IE even for diverging $\Delta(A)$ [4]. 

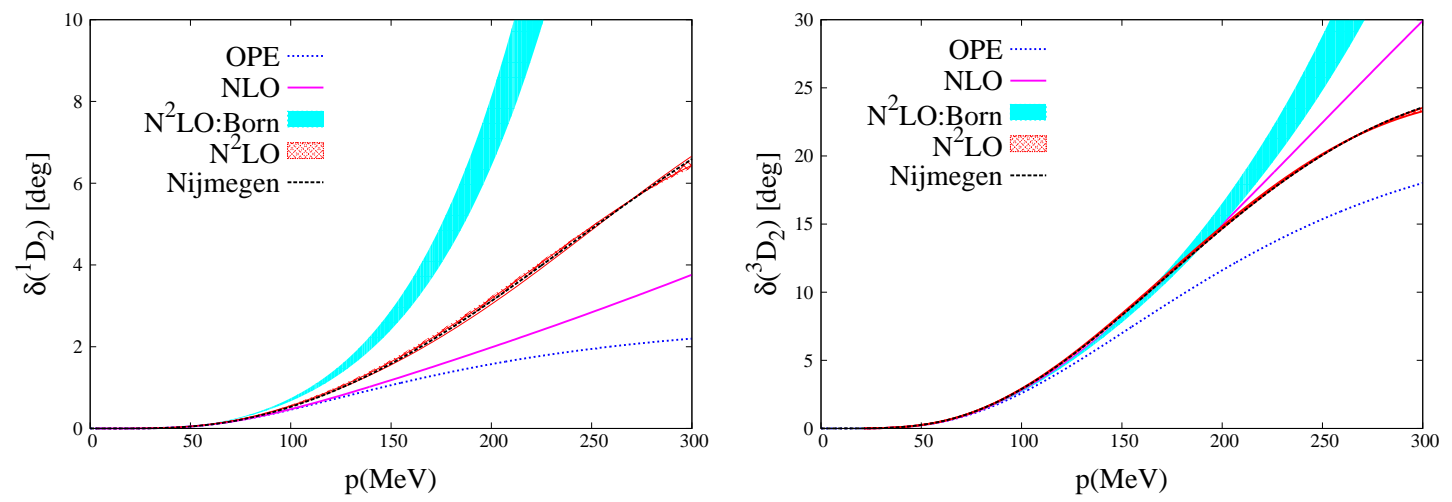

Figure 6: (Color online.) Phase shifts for ${ }^{1} D_{2}$ (left panel) and ${ }^{3} D_{2}$ (right panel). The (red) hatched areas correspond to the NNLO results while the (magenta) solid lines are the NLO outcome [44]. In both cases twice-subtracted DRs are used. The phase shifts in the Born approximation are shown by the (cyan) filled bands, the OPE result from Ref. [42] is the (blue) dotted lines and the Nijmegen PWA phase shifts are given by the (black) dashed lines.

$$
\begin{aligned}
& D(A)=1+\delta_{2} A+\frac{A^{2}}{\pi} \int_{-\infty}^{L} d k^{2} \frac{\Delta\left(k^{2}\right) D\left(k^{2}\right)}{\left(k^{2}\right)^{2}} g\left(A, k^{2}\right), \\
& N(A)=\frac{A^{2}}{\pi} \int_{-\infty}^{L} d k^{2} \frac{\Delta\left(k^{2}\right) D\left(k^{2}\right)}{\left(k^{2}-A\right)\left(k^{2}\right)^{2}} .
\end{aligned}
$$

The only free parameter per partial wave is $\delta_{2}=D^{(1)}(0)$ which is fitted to the Nijmegen PWA phase shifts. Taking into account the different sets of values for the $c_{i}$ counterterms we have the following results,

$$
\begin{aligned}
& { }^{1} D_{2}: D^{(1)}(0)=0.07(1) M_{\pi}^{-2}, \\
& { }^{3} D_{2}: D^{(1)}(0)=-0.017(3) M_{\pi}^{-2} .
\end{aligned}
$$

The reproduction of data is excellent as shown by the (red) hatched areas in Fig. 6, where the phase shifts for the ${ }^{1} D_{2}$ are given in the left panel and those of the ${ }^{3} D_{2}$ in the right one. Our results indeed overlap the Nijmegen PWA phase shifts given by the (black) dashed lines. Reference [44 obtained the (magenta) solid line making use also of twice-subtracted DRs at NLO. We see a remarkable improvement from NLO to NNLO due to the inclusion of NLO $\pi N$ dynamics, particularly for the ${ }^{1} D_{2}$ partial wave.

\subsection{Perturbative and Born approximation phase shifts}

The higher is the orbital angular momentum $\ell$ the more perturbative is expected to be the corresponding $N N$ partial wave. This statement was studied in detail in the perturbative study of Ref. [46] by making use of the one-loop approximation in BChPT. Indeed, we can easily obtain from our formalism both the leading perturbative solution to the IEs of the $N / D$ method in powers of $\Delta(A)$, as well as the leading term in the Born series approximation for the chiral $N N$ amplitude calculated up to $\mathcal{O}\left(p^{3}\right)$ in Ref. [46. The point is that for a weak interaction (small $\Delta(A)$ at low three-momentum) one can expect that $D(A) \simeq 1$ at low energies. It is then reasonable to consider that substituting $D(A) \rightarrow 1$ in the integral on the r.h.s. 
of Eq. (18) would be meaningful to calculate $N(A)$, because we have a rapid converging integral due to the factor $\left(k^{2}\right)^{\ell}$ in the denominator for a sufficiently large value of $\ell 15$ The perturbative result for $N(A)$, denoted by $N_{p}(A)$, is then

$$
N^{(p)}(A)=\frac{A^{\ell}}{\pi} \int_{-\infty}^{L} d k^{2} \frac{\Delta\left(k^{2}\right)}{\left(k^{2}\right)^{\ell}\left(k^{2}-A\right)}
$$

Had we included only the two-nucleon irreducible contributions to $\Delta(A)$, which is then denoted as $\Delta_{B}(A)$, the previous integral becomes the DR representation of the $N N$ potential that we denominate $N_{B}(A)$,

$$
N_{B}(A)=\frac{A^{\ell}}{\pi} \int_{-\infty}^{L} d k^{2} \frac{\Delta_{B}\left(k^{2}\right)}{\left(k^{2}\right)^{\ell}\left(k^{2}-A\right)} .
$$

This is due to the fact that the $N N$ potential projected in a given partial wave is an analytical function that only has LHC and it can be written in terms of a DR along the latter cut. We have checked numerically that the DR representation Eq. (57) for the $N N$ potential coincides with its explicit partial wave decomposition taking into account the expressions given in Ref. [46]. In our notation the relation between $N_{B}(A)$ and the phase shifts in the Born approximation, $\delta_{B}(A)$, reads

$$
\delta_{B}(A)=\rho(A) N_{B}(A) .
$$

An analogous expression holds for the perturbative phase shifts $\delta^{(p)}(A)$ calculated in terms of $N^{(p)}(A)$. The difference between the perturbative phase shifts and the Born approximation ones for $\ell \geq 2$ is typically not very significant and quite small. In the following we compare our full results with $\delta_{B}(A)$, since these phase shifts can be also calculated straightforwardly in potential models. We proceed in the same way for the coupled channel case as well by evaluating $N_{i j}(A)$ in the Born approximation by substituting $D_{i j}(A) \rightarrow 1$ in Eq. (21), and keeping only the two-nucleon irreducible contributions to $\Delta_{i j}(A)$.

Turning back to the uncoupled $D$ waves we also show in Fig. 6 the leading Born approximation phase shifts obtained from the NNLO two-nucleon irreducible contributions to $\Delta(A)$ by the (cyan) filled areas. One observes that these curves are quite different from our full results given by the hatched areas. This clearly indicates that the perturbative treatment of the $N N D$ waves is not accurate.

\section{Uncoupled $F$ waves}

For the $F$ waves we have three subtractions with two free parameters $\delta_{2}$ and $\delta_{3}$. We fix $\delta_{2}=0$ in the following (according to the principle of maximal smoothness) and fit $\delta_{3}$ to data. At NNLO the fitted values for $D^{(2)}(0)=2 \delta_{3}$, Eq. (19), are:

$$
\begin{aligned}
& { }^{1} F_{3}: \quad D^{(2)}(0)=0.057(3) M_{\pi}^{-4}, \\
& { }^{3} F_{3}: \quad D^{(2)}(0)=0.035(5) M_{\pi}^{-4},
\end{aligned}
$$

where the variation in the values is due to the different sets of $c_{i}$ counterterms employed. The NNLO results are shown by the (red) hatched areas in Fig. 7 which reproduce the Nijmegen PWA phase shifts (black dashed line) better than the NLO results (magenta lines) and the perturbative phase shifts (cyan filled areas). This improvement is particularly noticeable for the ${ }^{3} F_{3}$ partial wave.

\footnotetext{
${ }^{15}$ Of course, the precise meaning of this statement could vary from one case to the other due to characteristic facets of the considered partial wave.
} 

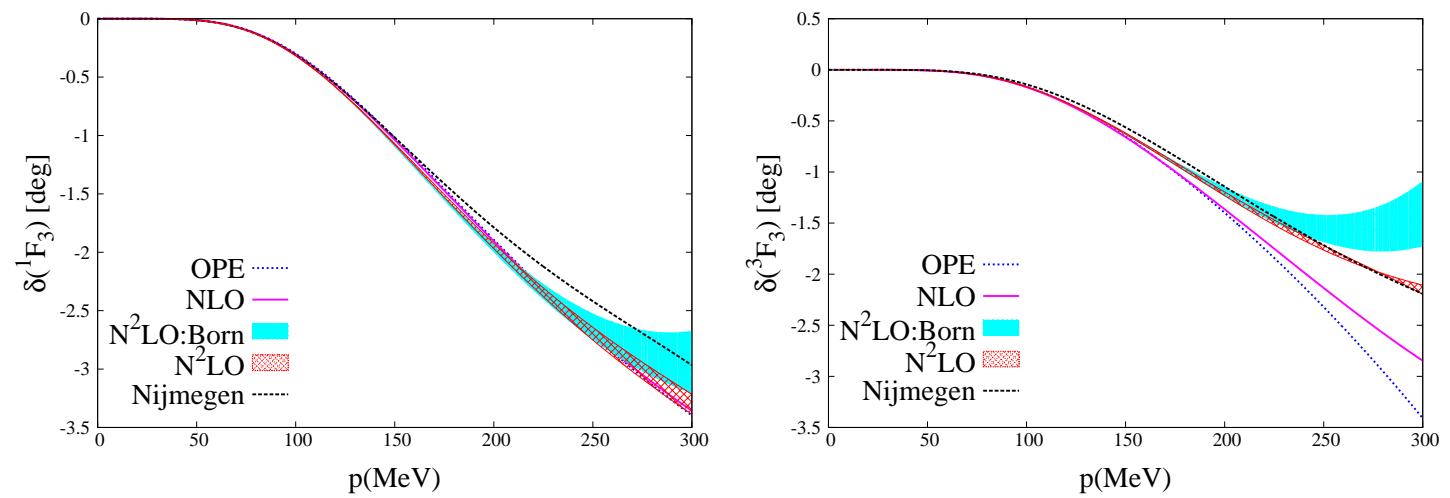

Figure 7: (Color online.) Phase shifts for ${ }^{1} F_{3}$ (left panel) and ${ }^{3} F_{3}$ (right panel). The (red) hatched areas correspond to the NNLO results while the (magenta) solid lines are the NLO outcome. In both cases three-time-subtracted DRs are used. The (cyan) filled bands give $\delta_{B}(A)$, the OPE result from Ref. [42] is the (blue) dotted lines and the Nijmegen PWA phase shifts correspond to the (black) dashed lines.

We also observe that for the $F$ waves the phase shifts in the leading Born approximation, Eq. (58), run much closer to our full results than for the $D$ waves, which clearly indicates that $F$ waves are more perturbative. Nevertheless, the relative deviation of the perturbation results compared with the full solution is still around a $50 \%$ at the end of the interval shown in Fig. 7 . A similar conclusion on the more perturbative nature of the $F$ waves was also reached in the pure perturbative study of Ref. [46] by comparing with experimental data. However, here we can also compare with the full unambiguous solution of the corresponding IE. For example, we can learn from Fig. 7 that the widths of the (cyan) filled bands for the Born approximation results reflect a much larger dependence on the $c_{i}$ coefficients than the one corresponding to the full nonperturbative results given by the (red) hatched areas. Thus, within our approach the failure reported in Refs. [32, 48] to reproduce simultaneously the $D$ and $F$ waves by using the NNLO chiral potential calculated in dimensional regularization in Ref. [46] because the large values of the $c_{i}$ counterterms does not happen. Namely, we are able to describe properly both the uncoupled $D$ and $F$ waves, Figs. 6] and 7, respectively, and the dependence on the precise set of $c_{i}$ 's taken is quite mild for the full results. Indeed our calculation at NNLO describe the Nijmegen PWA phase shifts better than the NLO ones [44], which is not the case for all of these waves in Ref. [32] based on the (modified) Weinberg approach when comparing their NLO and NNLO results.

The increase in the perturbative character of the $F$ waves can also be seen by considering the relevance of the different contributions of $\Delta(A)$ to the integral on the r.h.s. of Eq. (17), proceeding in a similar way to that already performed for the ${ }^{1} S_{0}$ partial wave in Sec. 4. The result is shown in the left panels of Fig. 8 , where the first row corresponds to ${ }^{1} F_{3}$ and the second to ${ }^{3} F_{3}$. In the right panels we show directly the different contributions to $\Delta(A)$. The meanings of the lines in Fig. 8 are the same as in Fig. 2 , though here the $c_{i}$ 's are taken from Ref. [11, given in the last row of Table 1, which is enough for the present purposes. Notice, that now a qualitative different situation is found with respect to what is shown in Fig. 2, that also holds for the $P$ and $D$ waves discussed in Secs. 5 and 6 . For the $F$ and higher waves the subleading two-nucleon irreducible TPE contribution is much less important and OPE is by far the dominant contribution, as it should correspond to a perturbative high- $\ell$ wave. 

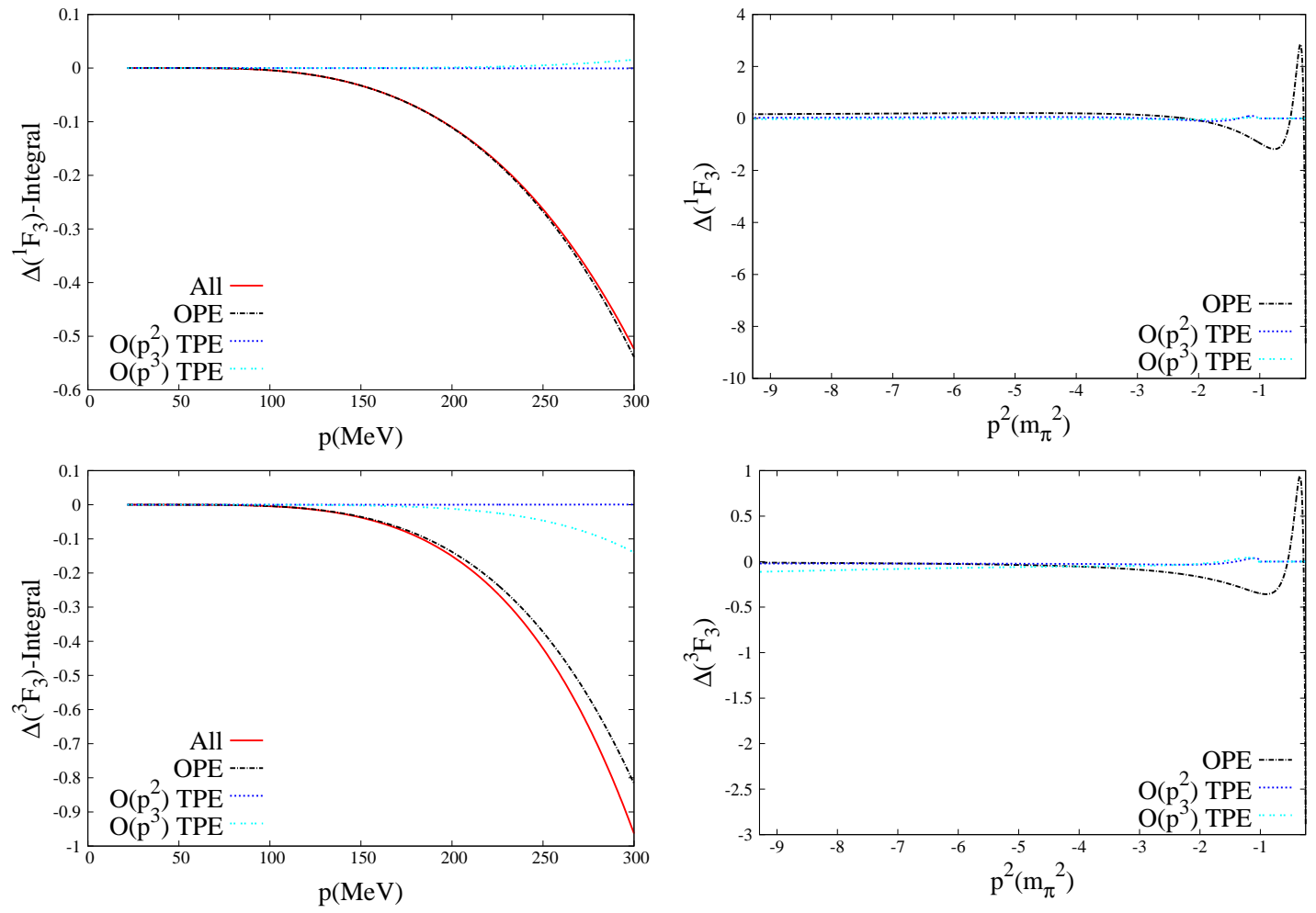

Figure 8: (Color online.) Left panel: different contributions to the integral on the r.h.s. of Eq. (17) for $\ell=3$. The meanings of the lines are the same as in Fig. 2. For definiteness we consider the $c_{i}$ 's given in the last row of Table 1. 

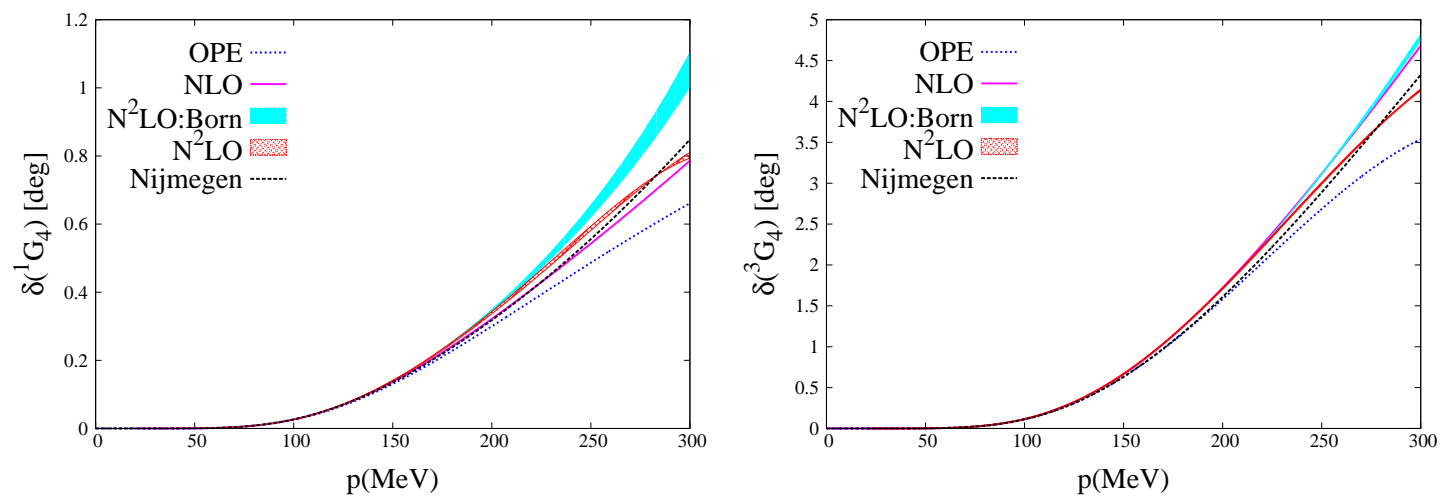

Figure 9: (Color online.) Phase shifts for ${ }^{1} G_{4}$ (left panel) and ${ }^{3} G_{4}$ (right panel). The (red) hatched areas correspond to the NNLO results while the (magenta) solid lines are the NLO outcome. In both cases four-time-subtracted DRs are used. The (cyan) filled areas represent the outcome from the leading Born approximation, the OPE result from Ref. [42] is the (blue) dotted lines and the Nijmegen PWA analysis is the (black) dashed lines.

\section{Uncoupled $G$ waves}

For the $G$ waves we have four subtractions of which $\delta_{i}(i=2,3,4)$ are free but, according to the principle of maximal smoothness, all of them are fixed to 0 except $\delta_{4}=D^{(3)}(0) / 3$ ! that is fitted to data. At NNLO the fitted values for $D^{(3)}(0)$ are:

$$
\begin{aligned}
& { }^{1} G_{4}: D^{(3)}(0)=-0.014(2) M_{\pi}^{-6}, \\
& { }^{3} G_{4}: D^{(3)}(0)=-0.055(5) M_{\pi}^{-6},
\end{aligned}
$$

where the variation in the values is due to the different sets of $c_{i}$ counterterms employed. The corresponding results are shown by the (red) hatched areas in Fig. 9. For both partial waves the actual dependence on the $c_{i}$ coefficients for the resulting phase shifts is almost negligible and the hatched areas degenerate to lines. The low-energy results are very similar at NLO and NNLO and reproduce the Nijmegen PWA phase shifts quite well. These results are better than the perturbative ones in the Born approximation, Eq. (58), which are shown by the (cyan) filled areas. As indicated for the uncoupled $F$ waves here OPE overwhelmingly dominates the different contribution to the dispersive integral on the r.h.s. of Eq. (17). This indicates that these waves are rather perturbative, though still we observe differences around $30 \%$ for $p \lesssim 300 \mathrm{MeV}$ in Fig. 9 between the full and perturbative results.

\section{Uncoupled $H$ waves}

For the case of the uncoupled $H$ waves, ${ }^{1} H_{5}$ and ${ }^{3} H_{5}$, we apply the five-time subtracted DRs of Eqs. (17) and (18) with $\ell=5$. We fit $\delta_{5}=D^{(4)}(0) / 4$ ! to the Nijmegen PWA phase shifts, which for $\ell \geq 5$ correspond to those obtained from the $N N$ potential model of Ref. [64], while $\delta_{2,3,4}$ are fixed to 0 (principle of maximal smoothness). We obtain the fitted values:

$$
\begin{aligned}
& { }^{1} H_{5}: \quad D^{(4)}(0)=0.156 M_{\pi}^{-8}, \\
& { }^{3} H_{5}: \quad D^{(4)}(0)=0.066 M_{\pi}^{-8} .
\end{aligned}
$$



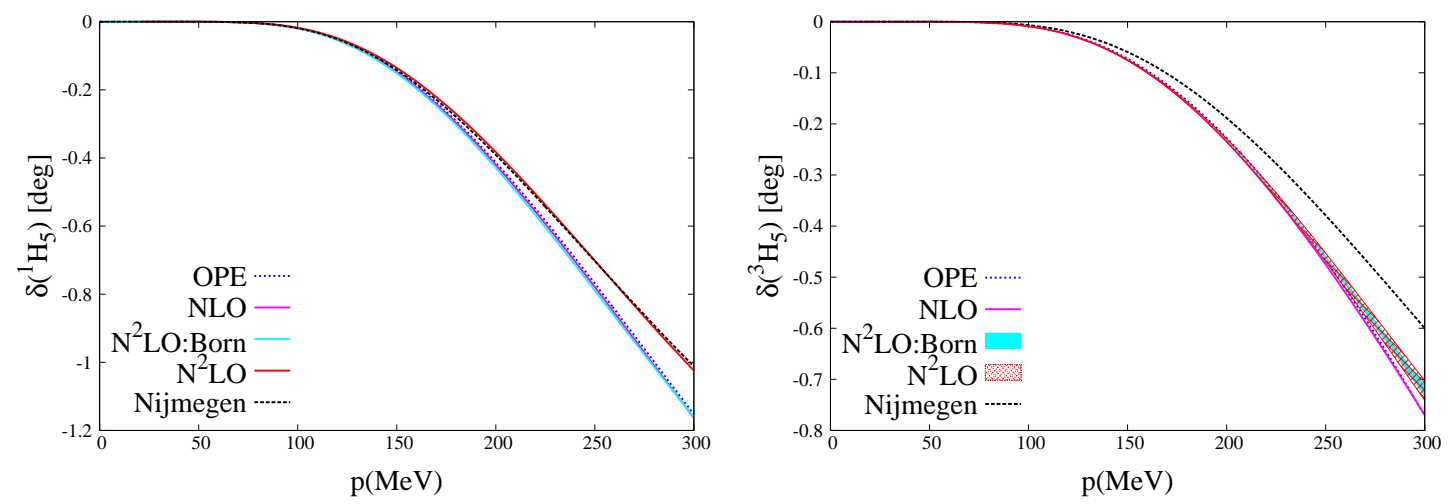

Figure 10: (Color online.) Phase shifts for ${ }^{1} H_{5}$ (left panel) and ${ }^{3} H_{5}$ (right panel). The (red) hatched areas correspond to the NNLO results while the (magenta) solid lines are the NLO outcome. The (cyan) filled bands correspond to $\delta_{B}(A)$, the OPE result from Ref. [42] is the (blue) dotted lines and the Nijmegen PWA is the (black) dashed lines.
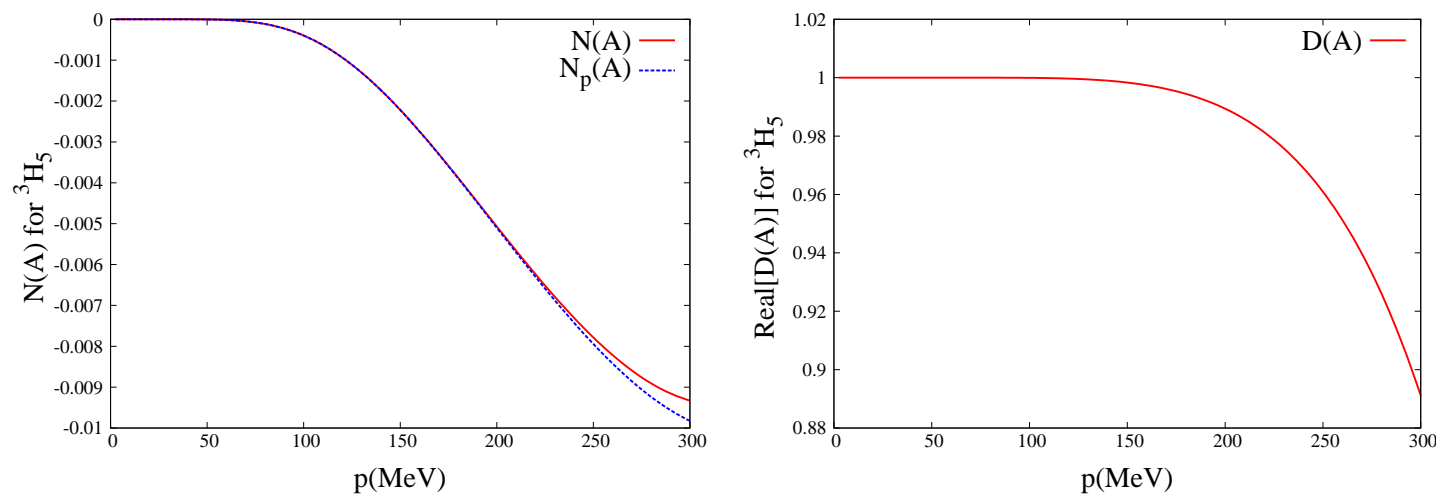

Figure 11: (Color online.) The functions $N(A)$ and $N_{p}(A)$ are shown by the (red) solid and (blue) dash-dotted lines in the left panel, respectively. The real part of the function $D(A)$ is plotted in the right panel.

The resulting fit is stable if we release $\delta_{i}(i=2,3,4)$. The phase shifts obtained are shown by the (red) hatched areas in Fig. 10 by taking into account the spread of the results depending of the set of $c_{i}$ 's chosen. In this figure the left panel corresponds to ${ }^{1} \mathrm{H}_{5}$ and the right one to ${ }^{3} \mathrm{H}_{5}$. For the former the resulting curve indeed overlaps the Nijmegen PWA phase shifts [45]. We also show by the (cyan) filled bands the phase shifts in the leading Born approximation which run rather close to the full results, indeed for the ${ }^{3} H_{5}$ case the (cyan) filled band is overlapped by the (red) hatched one. This clearly indicates the perturbative nature for the $H$ waves. For them it is also true that OPE overwhelmingly dominates the dispersive integral on the r.h.s. of Eq. (17), which is also the expected behavior for a perturbative partial wave. Notice that for the ${ }^{1} H_{5}$ wave the dependence on the actual values of the $c_{i}$ coefficients is so small that at the end the hatched and filled areas collapse to lines. For the ${ }^{3} H_{5}$ case there is a visible, albeit small, dependence on the set of $c_{i}$ 's employed. In both cases the NNLO results reproduce the Nijmegen PWA phase shifts closer than the NLO and OPE results.

It is interesting to discuss in this case the behavior of the function $N(A)$ compared with $N_{p}(A)$, given 
in Eq. (56). The main point is that here both $N(A)$ and $D(A)$ have a zero at around $450 \mathrm{MeV}$. We consider only the ${ }^{3} H_{5}$ wave because a similar discussion would follow for ${ }^{1} \mathrm{H}_{5}$ as well, that we skip for brevity. In the left panel of Fig. 11] we show by the (red) solid line the full $N(A)$ and by the (blue) dashed line the perturbative result $N_{p}(A)$. We see that they are very similar, as expected for a partial wave with an $\ell$ as high as 5. In addition, we display in the right panel of the same figure the real part of $D(A)$ from Eq. (17), which is very close to 1 , as expected for a situation with a weak interaction as well. All these curves are obtained by employing the $c_{i}$ 's from Ref. [11]. A bit higher in energy both $N_{p}(A)$ and $N(A)$ have a zero at around $\sqrt{A}=450 \mathrm{MeV}$. Since $T(A)=N(A) / D(A)$ this would imply that $T(A)=0$ at that energy, which is at odds with the values of the phase shifts given by the Nijmegen PWA [45] that do not vanish at this point. The only remedy is that $D(A)$ is also zero at the same point so that one had a limit $0 / 0$ that is finally finite. This is indeed the case and it is is the reason why $D(A)$ starts to decrease for $\sqrt{A}>200 \mathrm{MeV}$ in Fig. [11,

Another question of interest to think about is what have we gained by solving exactly Eq. (17) instead of using only the perturbative solution, Eq. (56) , or the Born approximation, Eq. (57), with the related $\delta_{B}(A)$, Eq. (58)?. The main point that one should consider in connection with this question is that by solving the full and nonperturbative Eq. (17) (furthermore, in good agreement with data) one can then state that Eq. (56) is a perturbation of a well-defined and existing nonperturbative solution. By solving exactly Eq. (17) we have needed to consider explicitly $\delta_{5}$ as a free parameter for the uncoupled $H$ waves and fit it to the Nijmegen PWA. Indeed, $\delta_{5}$ is not only necessary for a good fit, but it is also required in order to keep $D(A) \simeq 1$ at low three-momentum. Otherwise, the contribution from the dispersive integral to $D(A)$ on the r.h.s. of Eq. (18) would be too large and negative and would render a too strong function $N(A)$ in plain disagreement with $N_{p}(A)$. Notice as well that in the case of the partial wave ${ }^{1} H_{5}$ a better reproduction of data is achieved than with $\delta_{B}(A)$. It is also worth recalling the previous finding in Sec. 7 for the $F$ waves, where the full results show a much smaller dependence on the set of $c_{i}$ coefficients used than the perturbative or Born approximation phase shifts, cf. Fig. 7.

\section{Coupled ${ }^{3} S_{1}-{ }^{3} D_{1}$ waves}

We start our study of the ${ }^{3} S_{1}-{ }^{3} D_{1}$ coupled-partial-wave system in terms of just one free parameter, that we choose as the pole position of the deuteron in the $A$-complex plane, $k_{d}^{2}=-m E_{d}$, with $E_{d}=2.225 \mathrm{MeV}$ the deuteron binding energy. Thus we implement once-subtracted DRs for the ${ }^{3} S_{1}$ and twice-subtracted ones for the ${ }^{3} D_{1}$. In the case of the mixing partial wave we have a mixed situation with a once-subtracted DR for $N_{12}(A)$ and a twice-subtracted one for $D_{12}(A)$. In this way we guarantee both the right threshold behavior as well as the experimental deuteron-pole position in all the partial waves. We write now explicitly the DRs considered. For the ${ }^{3} S_{1}$ one has,

$$
\begin{aligned}
& D_{11}(A)=1-\frac{A}{k_{d}^{2}} \frac{g_{11}(A, 0)}{g_{11}\left(k_{d}^{2}, 0\right)}+\frac{A}{\pi} \int_{-\infty}^{L} d k^{2} \frac{\Delta_{11}\left(k^{2}\right) D_{11}\left(k^{2}\right)}{k^{2}}\left[g_{11}\left(A, k^{2}\right)-g_{11}(A, 0) \frac{g_{11}\left(k_{d}^{2}, k^{2}\right)}{g_{11}\left(k_{d}^{2}, 0\right)}\right], \\
& N_{11}(A)=\nu_{1}^{(11)}+\frac{A}{\pi} \int_{-\infty}^{L} d k^{2} \frac{\Delta_{11}\left(k^{2}\right) D_{11}\left(k^{2}\right)}{k^{2}\left(k^{2}-A\right)},
\end{aligned}
$$

with all the subtractions taken at $A=0$ and the new function $g_{i j}(A)$ is defined as

$$
g_{i j}\left(A, k^{2}\right)=\frac{1}{\pi} \int_{0}^{\infty} d q^{2} \frac{\nu_{i j}\left(q^{2}\right)}{\left(q^{2}-A\right)\left(q^{2}-k^{2}\right)},
$$


The subtraction constant $\nu_{1}$ in $N_{11}(A)$ is fixed by imposing that $D_{11}\left(k_{d}^{2}\right)=0$,

$$
\nu_{1}^{(11)}=\frac{1}{k_{d}^{2} g_{11}\left(k_{d}^{2}, 0\right)}\left[1+\frac{k_{d}^{2}}{\pi} \int_{-\infty}^{L} d k^{2} \frac{\Delta_{11}\left(k^{2}\right) D_{11}\left(k^{2}\right)}{k^{2}} g_{11}\left(k^{2}, k_{d}^{2}\right)\right]
$$

a result that is already implemented in Eq. (62) for $D_{11}(A)$.

The corresponding DRs for the ${ }^{3} D_{1}$ and the mixing wave can be grouped together in the same form,

$$
\begin{aligned}
& D_{i j}(A)=1-\frac{A}{k_{d}^{2}}+\frac{A\left(A-k_{d}^{2}\right)}{\pi} \int_{-\infty}^{L} d k^{2} \frac{\Delta_{i j}\left(k^{2}\right) D_{i j}\left(k^{2}\right)}{\left(k^{2}\right)^{\ell_{i j}}} g_{i j}^{(d)}\left(A, k^{2} ; \ell_{i j}\right), \\
& N_{i j}(A)=\frac{A^{\ell_{i j}}}{\pi} \int_{-\infty}^{L} d k^{2} \frac{\Delta_{i j}\left(k^{2}\right) D_{i j}\left(k^{2}\right)}{\left(k^{2}\right)^{\ell_{i j}}\left(k^{2}-A\right)} .
\end{aligned}
$$

where $\ell_{12}=1$ and $\ell_{22}=2$ and all the subtractions for the $N_{i j}(A)$ are taken at $A=0$, while in the function $D(A)$ one is taken at $A=0$ and the other at $A=k_{d}^{2}$. The function $g_{i j}^{(d)}\left(A, k^{2} ; m\right)$ is defined as

$$
g_{i j}^{(d)}\left(A, k^{2} ; m\right)=\frac{1}{\pi} \int_{0}^{\infty} d q^{2} \frac{\nu_{i j}\left(q^{2}\right)\left(q^{2}\right)^{m-1}}{\left(q^{2}-A\right)\left(q^{2}-k^{2}\right)\left(q^{2}-k_{d}^{2}\right)} .
$$

The results obtained by solving the IEs for the functions $D_{i j}(A)$ along the LHC from Eqs. (62) and (65) are shown in Fig. 12 by the (cyan) filled areas. These results are indicated as NNLO-I and all the subtraction constants are fixed in terms of $k_{d}^{2}$, without any other freedom. The spread in the results originates by taking different sets of $c_{i}$ 's from Refs. [11,55] and varying the input in the iterative procedure. The present NNLO calculation from Eqs. (62) and (65) reproduces the Nijmegen PWA mixing angle $\epsilon_{1}$ much better than the NLO result from the same set of equations, which is shown by the (magenta) dot-dashed lines. This improvement in the description of $\epsilon_{1}$ when passing from NLO to NNLO is also seen in Ref. 32 by employing the Weinberg scheme. The ${ }^{3} S_{1}$ phase shifts are also reproduced better at NNLO than at NLO, while the ${ }^{3} D_{1}$ phase shifts are somewhat worse described by the former. Our results for the ${ }^{3} S_{1}$ and ${ }^{3} D_{1}$ phase shifts are quite similar to those obtained in Ref. [34, but not for $\epsilon_{1}$ where our outcome is closer to the Nijmegen PWA. The comparison is not so straightforward with the results of Ref. [37, which depend very much on the type of chiral $N N$ potential used. For the ${ }^{3} S_{1}-{ }^{3} D_{1}$ coupled partial waves we do not show the Born approximation results in Fig. 12 because they are specially poor, see e.g. Refs. [46, 48] for the ${ }^{3} D_{1}$ phase shifts.

We can also predict from Eqs.(62) and (65) the ${ }^{3} S_{1}$ scattering length $\left(a_{t}\right)$ and effective range $\left(r_{t}\right)$. The former is given in terms of $\nu_{1}^{(11)}$, Eq. (64), as

$$
a_{t}=-\frac{m \nu_{1}^{(11)}}{4 \pi} .
$$

Regarding $r_{t}$ we can proceed similarly as discussed in detail in Ref. [44] where the following expression is derived,

$$
r_{t}=-\frac{m}{2 \pi^{2} a_{t}} \int_{-\infty}^{L} d k^{2} \frac{\Delta_{11}\left(k^{2}\right) D_{11}\left(k^{2}\right)}{\left(k^{2}\right)^{2}}\left\{\frac{1}{a_{t}}+\frac{4 \pi k^{2}}{m} g_{11}\left(0, k^{2}\right)\right\}-\frac{8}{m} \int_{0}^{\infty} d q^{2} \frac{\nu_{11}\left(q^{2}\right)-\rho\left(q^{2}\right)}{\left(q^{2}\right)^{2}},
$$

This equation also exhibits a correlation between $r_{t}$ and $a_{t}$, although in a more complicated manner than for the ${ }^{1} S_{0}$ partial wave, as shown in Eq. (36), because $\nu_{11}(A)$ depends nonlinearly on $D_{11}(A)$. 

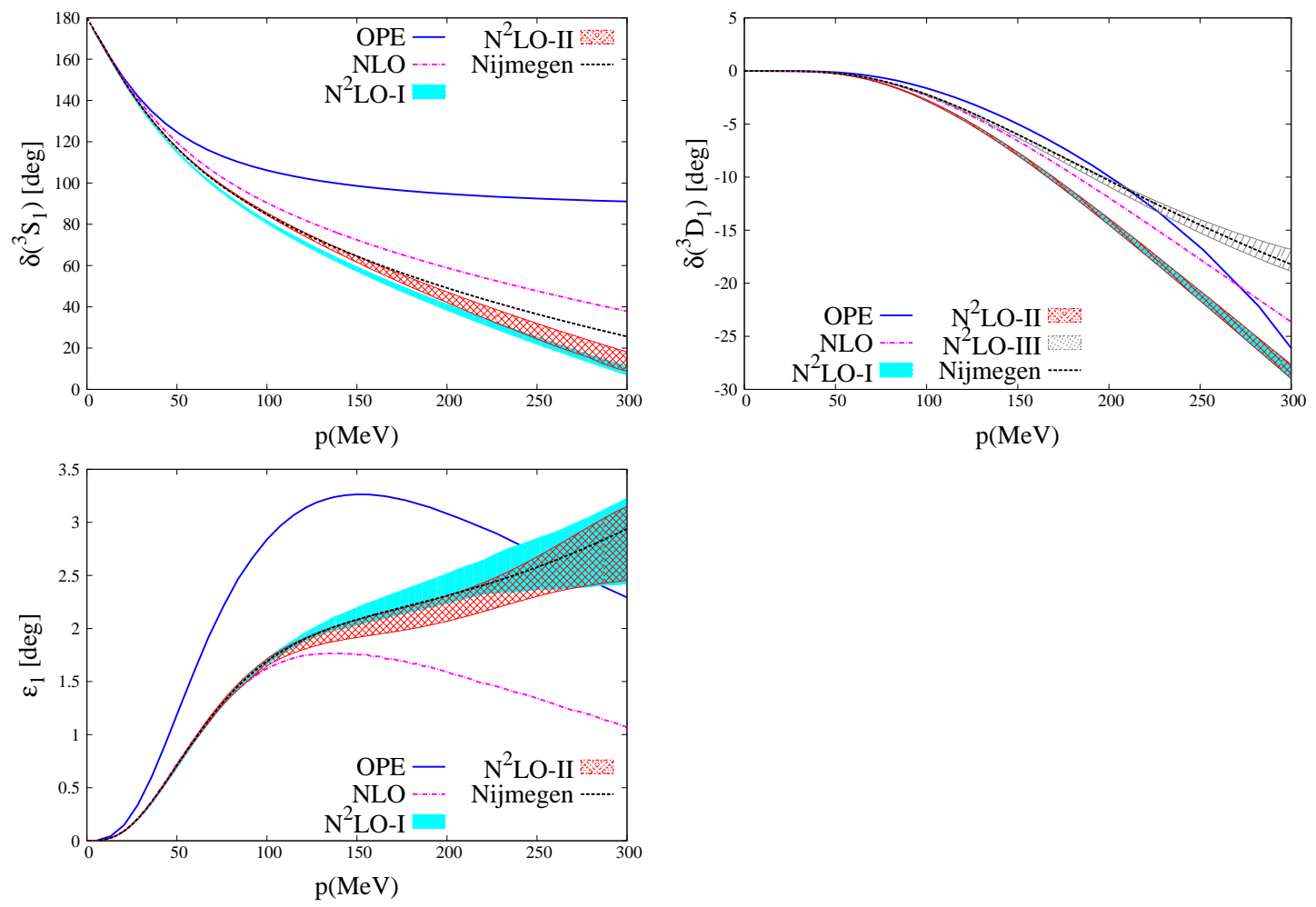

Figure 12: (Color online.) From left to right and top to bottom: Phase shifts for ${ }^{3} S_{1},{ }^{3} D_{1}$ and the mixing angle $\epsilon_{1}$, respectively. The (cyan) filled areas correspond to the NNLO-I outcome obtained by solving Eqs. (62) and (65)). The hatched areas with (red) crossed lines are the NNLO-II results that stem from Eqs. (76), (77) and (65). In addition, for the ${ }^{3} D_{1}$ we show by the hatched areas with (gray) parallel lines the results obtained by employing three-time subtracted DRs for ${ }^{3} D_{1}$, Eq. (779). As usual, the (magenta) dot-dashed lines are the NLO phase shifts and mixing angle, the LO ones are given by the (blue) dotted lines and the Nijmegen PWA results correspond to the (black) dashed lines.

Another observable that we also consider is the slope at threshold of $\epsilon_{1}$, indicated as $a_{\varepsilon}$, and defined by

$$
a_{\varepsilon}=\lim _{A \rightarrow 0^{+}} \frac{\sin 2 \epsilon_{1}}{A^{\frac{3}{2}}}=1.128 M_{\pi}^{-3}
$$

where the numerical value is deduced from the Nijmegen PWA phase shifts. From the DRs in Eq. (65) we obtain the following expression for $a_{\varepsilon}$,

$$
a_{\varepsilon}=\frac{m}{4 \pi^{2}} \int_{-\infty}^{L} d k^{2} \frac{\Delta_{i j}\left(k^{2}\right) D_{i j}\left(k^{2}\right)}{\left(k^{2}\right)^{2}} .
$$

It is also interesting to diagonalize the ${ }^{3} S_{1}-{ }^{3} D_{1} S$-matrix around the deuteron pole position. This can be done by means of a real orthogonal matrix [65],

$$
\mathcal{O}=\left(\begin{array}{ll}
\cos \varepsilon_{1} & -\sin \varepsilon_{1} \\
\sin \varepsilon_{1} & \cos \varepsilon_{1}
\end{array}\right)
$$




\begin{tabular}{|l|l|l|l|l|l|}
\hline & $a_{t}[\mathrm{fm}]$ & $r_{t}[\mathrm{fm}]$ & $\eta$ & $N_{p}^{2}\left[\mathrm{fm}^{-1}\right]$ & $a_{\varepsilon}\left[M_{\pi}^{-3}\right]$ \\
\hline NLO & 5.22 & 1.47 & 0.0295 & 0.714 & 1.372 \\
\hline NNLO-I & $5.52(3)$ & $1.89(3)$ & $0.0242(3)$ & $0.818(10)$ & $1.270(9)$ \\
\hline NNLO-II & $5.5424^{\star}$ & $1.759^{\star}$ & $0.02535(13)$ & $0.78173(2)$ & $1.293(8)$ \\
\hline Ref. [65] & $5.4194(20)$ & $1.7536(25)$ & $0.0253(2)$ & $0.7830(15)$ & \\
\hline Ref. [32] & 5.424 & 1.753 & 0.0245 & & \\
\hline
\end{tabular}

Table 4: Values for $a_{t}, r_{t}, \eta, N_{p}^{2}$ and $a_{\varepsilon}$. The results predicted from Eqs. (62) and (65) are given in the second (NLO) and third row (NNLO-I). The values given in the fourth row (NNLO-II) are obtained once $a_{t}$ and $r_{t}$ are fixed to the experimental figures, which is indicated by a star on top of the values. We also show the results from Refs. [65] and [32] in the fifth and sixth rows, respectively.

Such that

$$
S=\mathcal{O}\left(\begin{array}{ll}
S_{0} & 0 \\
0 & S_{2}
\end{array}\right) \mathcal{O}^{T}
$$

with $S_{0}$ and $S_{2}$ the $S$-matrix eigenvalues. The asymptotic $D / S$ ratio of the deuteron, $\eta$, can be expressed in terms of $\varepsilon_{1}$ as

$$
\eta=-\tan \varepsilon_{1}
$$

The residue of $S_{0}$ at the deuteron pole position is denoted by $N_{p}^{2}$,

$$
N_{p}^{2}=\lim _{A \rightarrow k_{d}^{2}}\left(\sqrt{-k_{d}^{2}}+i \sqrt{A}\right) S_{0}
$$

As discussed in Ref. 66 the shape parameters are a good testing ground for the range of applicability of the underlying EFT. We then study our results for the shape parameters of the lowest eigenphase $\delta_{0}$ (also called ${ }^{3} S_{1}$ eigenphase), Eq. (72), with the diagonalization of the $S$-matrix performed in the physical region $A \geq 0$

$$
\sqrt{A} \cot \delta_{0}=-\frac{1}{a_{t}}+\frac{1}{2} r_{t} A+\sum_{i=2}^{10} v_{i} A^{i}+\mathcal{O}\left(A^{11}\right) .
$$

The scattering length and effective range in the previous equation are the same as given above because coupled-wave effects with the ${ }^{3} D_{1}$ only affects the shape parameters $v_{i}, i \geq 2$. The values obtained at NLO and NNLO from Eqs. (62) and (65) for $a_{t}, r_{t}, \eta, N_{p}^{2}$ and $a_{\varepsilon}$ are shown in Table 4 in the second and third rows, respectively. We observe that the numbers at NNLO (indicated by NNLO-I) are already rather close to those of Ref. [65], obtained from the Nijmegen PWA of $n p$ data, and Ref. [32]. It is interesting to remark that our value for $r_{t}$ is a prediction in terms of only one subtraction constant (fixed by the deuteron pole position) and $N N$ forces stemming from $\pi N$ physics. This value deviates from experiment $r_{t}=1.759 \pm 0.005 \mathrm{fm}[32$ around a $10 \%$ at $\mathrm{NNLO}(\sim 20 \%$ at NLO$)$, while the relative experimental error is around $3 \%$. Other determinations for the parameter $\eta$, not shown in Table 4, are $\eta=0.0256(4)$ [67], $\eta=0.0271(4)$ [68], $\eta=0.0263(13)$ [69] and $\eta=0.0268(7)$ [70].

\footnotetext{
${ }^{16}$ This can also be done in terms of an orthogonal matrix Eq. (71) because of two-body unitarity.
} 


\begin{tabular}{|l|l|l|l|l|l|}
\hline & $v_{2}$ & $v_{3}$ & $v_{4}$ & $v_{5}$ & $v_{6}$ \\
\hline NLO & $-0.10572(12)$ & $0.8818(11)$ & $-5.427(11)$ & $36.73(11)$ & $-259.9(1.1)$ \\
\hline NNLO-I & $0.157(22)$ & $0.645(9)$ & $-3.41(13)$ & $23.2(8)$ & $-161(6)$ \\
\hline NNLO-II & $0.0848(4)$ & $0.762(7)$ & $-4.33(2)$ & $29.0(2)$ & $-198(2)$ \\
\hline Ref. [65] & $0.040(7)$ & $0.673(2)$ & $-3.95(5)$ & $27.0(3)$ & \\
\hline Ref. [32] & 0.046 & 0.67 & -3.9 & & \\
\hline
\end{tabular}

Table 5: Values for the shape parameters $v_{i}, i=2, \ldots, 6$ in units of $\mathrm{fm}^{2 i-1}$. The results predicted from Eqs. (62) and (65) are given in the second (NLO) and third row (NNLO-I). The errors for the NLO results correspond entirely to the numerical accuracy in the calculation. Those values corresponding to NNLO-II are given in the fourth row. The values from Refs. [65] and [32] appear in the fifth and sixth rows, in order.

\begin{tabular}{|l|l|l|l|l|}
\hline & $v_{7}$ & $v_{8} \times 10^{-1}$ & $v_{9} \times 10^{-2}$ & $v_{10} \times 10^{-3}$ \\
\hline NLO & $1867(11)$ & $-1375(11)$ & $1008(11)$ & $-760(12)$ \\
\hline NNLO-I & $1161(41)$ & $-840(30)$ & $625(22)$ & $-463(17)$ \\
\hline NNLO-II & $1426(13)$ & $-1015(15)$ & $764(17)$ & $-545(20)$ \\
\hline
\end{tabular}

Table 6: Values for the shape parameters $v_{i}, i=7, \ldots, 10$ in units of $\mathrm{fm}^{2 i-1}$. For the meanings of the rows see Table 5 ,

The values for the shape parameters $v_{i}, i=2, \ldots, 6$, are given in Table 5 and for $i=7, \ldots, 10$ in Table 6. Up to our knowledge the values of the shape parameters with $i>5$ were not given before. We detailed in Appendix A the numerical method that allows us to perform the appropriate derivatives up to so high order 17 We could have also given shape parameters of even higher orders within a numerical precision of a few per cent, but this is skipped because its apparent little relevance in practice. One can appreciate the numerical precision in the calculation of the shape parameters by considering the errors in Tables 5 and 6 for the NLO results, which entirely correspond to the numerical accuracy. Notice that for the highest shape parameter shown, $v_{10}$, its relative error is $1.5 \%$, just slightly worse than for $v_{9}$ with a relative error of $1.1 \%$. We then see that by increasing the order of the shape parameter the numerical accuracy only worsens little by little. Morever, the errors at NNLO take into account additionally the variation in the results from the different sets of $c_{i}$ 's employed and the dependence in the input for starting the iterative process. For the shape parameters with large order, $i \geq 5$, their absolute values increase typically as $\mathcal{O}\left(1 / M_{\pi}\right)^{2 i-1}$, which is the expected behavior for long-range interactions mediated by OPE. It is clear from Table 5 that the shape parameters $v_{i}, i=2, \ldots, 5$ predicted by the NNLO-I calculation (third row) are typically closer to the values of Refs. [32,65] than those at NLO (second row). This is a positive feature indicating a well-behaved expansion of the results obtained by applying the $N / D$ method with the discontinuity $\Delta(A)$ expanded in BChPT.

According to the power counting for the subtraction constants, Eq. (28), at NNLO it is appropriate to consider twice-subtracted DRs. For the ${ }^{3} S_{1}-{ }^{3} D_{1}$ system this implies to take into account two more free parameters for the ${ }^{3} S_{1}$ wave and one more for the mixing partial wave. The three parameters for the ${ }^{3} S_{1}$ wave are fixed in terms of the experimental values of $k_{d}^{2}, r_{t}$ and $a_{t}$. The DR for the ${ }^{3} D_{1}$ wave is

\footnotetext{
${ }^{17}$ For example in Ref. 65] it is stated that their numerical set up is not precise enough to calculate $v_{6}$ and that it already casts doubts about the numerical accuracy for $v_{5}$.
} 
the same as in Eq. (65). The twice-subtracted DRs taken now regarding the ${ }^{3} S_{1}$ partial wave are

$$
\begin{aligned}
D_{11}(A) & =1-\frac{A}{k_{d}^{2}}-\nu_{1}^{(11)} A\left(A-k_{d}^{2}\right) g_{11}^{(d)}(A, 0 ; 1)-\nu_{2}^{(11)} A\left(A-k_{d}^{2}\right) g_{11}\left(A, k_{d}^{2}\right) \\
& +\frac{A\left(A-k_{d}^{2}\right)}{\pi} \int_{-\infty}^{L} d k^{2} \frac{\Delta_{11}\left(k^{2}\right) D_{11}\left(k^{2}\right)}{\left(k^{2}\right)^{2}} g_{11}^{(d)}\left(A, k^{2} ; 2\right), \\
N_{11}(A) & =\nu_{1}^{(11)}+\nu_{2}^{(11)} A+\frac{A^{2}}{\pi} \int_{-\infty}^{L} d k^{2} \frac{\Delta_{11}\left(k^{2}\right) D_{11}\left(k^{2}\right)}{\left(k^{2}\right)^{2}\left(k^{2}-A\right)}, \\
\nu_{1}^{(11)} & =-\frac{4 \pi a_{t}}{m}, \\
\nu_{2}^{(11)} & =\frac{\nu_{1}^{(11)}}{\nu_{1}^{(11)} k_{d}^{2} g_{11}\left(0, k_{d}^{2}\right)-1}\left\{\frac{1}{k_{d}^{2}}+a_{t}\left(\frac{4 k_{d}^{2}}{m} \int_{0}^{\infty} d q^{2} \frac{\nu_{11}\left(q^{2}\right)-\rho\left(q^{2}\right)}{\left(q^{2}\right)^{2}\left(q^{2}-k_{d}^{2}\right)}+\frac{1}{\sqrt{-k_{d}^{2}}}-\frac{r_{t}}{2}\right)\right. \\
& \left.+\frac{k_{d}^{2}}{\pi} \int_{-\infty}^{L} d k^{2} \frac{\Delta_{11}\left(k^{2}\right) D_{11}\left(k^{2}\right)}{\left(k^{2}\right)^{2}} g_{11}\left(k_{d}^{2}, k^{2}\right)\right\} .
\end{aligned}
$$

For the mixing partial wave the DRs are

$$
\begin{aligned}
& D_{12}(A)=1-\frac{A}{k_{d}^{2}}-\nu_{2}^{(12)} A\left(A-k_{d}^{2}\right) g_{12}\left(A, k_{d}^{2}\right)+\frac{A\left(A-k_{d}^{2}\right)}{\pi} \int_{-\infty}^{L} d k^{2} \frac{\Delta_{12}\left(k^{2}\right) D_{12}\left(k^{2}\right)}{\left(k^{2}\right)^{2}} g_{12}^{(d)}\left(A, k^{2} ; 2\right), \\
& N_{12}(A)=\nu_{2}^{(12)} A+\frac{A^{2}}{\pi} \int_{-\infty}^{L} d k^{2} \frac{\Delta_{12}\left(k^{2}\right) D_{12}\left(k^{2}\right)}{\left(k^{2}\right)^{2}\left(k^{2}-A\right)},
\end{aligned}
$$

The results obtained by solving the IEs of Eqs. (76), (77) and Eq. (65) with $\ell_{22}=2$ are denoted in the following by NNLO-II and correspond to the (red) hatched areas with crossed lines in Fig. 12, It turns out that we cannot obtain a solution of the resulting IE for $D_{12}(A)$ by implementing any arbitrary value for $\nu_{2}^{(12)}$. We have further checked this statement by employing the following expression for $\nu_{2}^{(12)}$,

$$
\nu_{2}^{(12)}=\frac{\Theta}{2 \pi} \int_{-\infty}^{L} d k^{2} \frac{\Delta_{i j}\left(k^{2}\right) D_{i j}\left(k^{2}\right)}{\left(k^{2}\right)^{2}} .
$$

Here, the integral is the same as in Eq. (70), so that if we take $\Theta=1$ we would simply rewrite the IE of Eq. (65) in terms of twice-subtracted DRs. Then, we vary $\Theta$ and whenever we find a meaningful solution the obtained value for $a_{\varepsilon}=m \nu_{2}^{(12)} / 2 \pi$ is always basically the same, $a_{\varepsilon} \simeq 1.30 M_{\pi}^{-3}$. In our opinion this difficulty in our approach to reproduce the value for $a_{\varepsilon}$ that follows from the Nijmegen PWA, Eq. (69), casts doubts on this number. Notice that the calculated values for $\epsilon_{1}$ at low momentum, e.g. for $\sqrt{A} \lesssim 100 \mathrm{MeV}$, lie on top of the curve for the Nijmegen PWA results as shown in the third panel of Fig. 12 by the coincident hatched and filled areas that overlap the Nijmegen PWA line. The phase shifts and $\epsilon_{1}$ are quite similar to the NNLO-I results in terms of just one free parameter. Nevertheless, the ${ }^{3} S_{1}$ phase shifts for NNLO-II are closer to the Nijmegen PWA ones at lower energies, but the change for this S-wave by going from once- to twice-subtracted DRs is much less notorious than in the case of the partial wave ${ }^{1} S_{0}$, discussed in Sec. 4. We can also see in the fourth row of Table 4 that the NNLO-II values for $\eta$ and $N_{p}^{2}$ are compatible with those of Ref. [65, which is quite remarkable. The value for $a_{\varepsilon}$ mentioned above is shown in the last column of the same table. The shape parameters are shown in the forth rows of Tables [5] and 6, where we observe a better agreement with the numbers given in Ref. 65] for $v_{4}$ and $v_{5}$ than for $v_{2}$ and $v_{3}$. The variation of the values between NNLO-I and NNLO-II for the higher order shape parameters allows us to guess in a conservative way the systematic uncertainty affecting their calculation. 
On the other hand, we would like to elaborate further on the fact that at NNLO the results for the ${ }^{3} D_{1}$ phase shifts do not still offer a good reproduction of the Nijmegen PWA ones, being even worse than those obtained at NLO. In Ref. [48] one can find a discussion on the difficulties arisen in their calculation because of the large values of the NLO $\pi N$ counterterms, namely $c_{3}$ and $c_{4}$, in order to reproduce simultaneously the $D$ and $F$ waves within the Weinberg scheme using the NNLO chiral potential calculated in dimensional regularization. Considering this observation we obtain that when all the $c_{i}=0$ our NNLO result for $\delta_{2}$ is then essentially the same as the NLO one in Fig. 12, corresponding to the (magenta) dot-dashed line. In view of this, we study now the influence in the results by including one more subtraction in the DRs for ${ }^{3} D_{1}$ with the aim of determining whether this worsening is an effect that can be counterbalanced in a natural way at $\mathcal{O}\left(p^{4}\right)$. In this way we use the same twice-subtracted DRs for ${ }^{3} S_{1}$ and the mixing partial wave given in Eqs. (76) and (77), respectively, while the following three-time subtracted DRs are used for the ${ }^{3} D_{1}$

$$
\begin{aligned}
D_{22}(A) & =1-\frac{A}{k_{d}^{2}}+\delta_{3}^{(22)} A\left(A-k_{d}^{2}\right)-\nu_{3}^{(22)} A\left(A-k_{d}^{2}\right)^{2} \frac{\partial g_{22}^{(d)}(A, 0 ; 2)}{\partial k_{d}^{2}} \\
& +\frac{A\left(A-k_{d}^{2}\right)^{2}}{\pi} \int_{-\infty}^{L} d k^{2} \frac{\Delta_{22}\left(k^{2}\right) D_{22}\left(k^{2}\right)}{\left(k^{2}\right)^{3}} \frac{\partial g_{22}^{(d)}\left(A, k^{2} ; 3\right)}{\partial k_{d}^{2}}, \\
N_{22}(A) & =\nu_{3}^{(22)} A^{2}+\frac{A^{3}}{\pi} \int_{-\infty}^{L} d k^{2} \frac{\Delta_{22}\left(k^{2}\right) D_{22}\left(k^{2}\right)}{\left(k^{2}\right)^{3}\left(k^{2}-A\right)}
\end{aligned}
$$

with two additional subtraction constants $\delta_{3}^{(22)}$ and $\nu_{3}^{(22)}$. Considering the results obtained from the twice-subtracted DRs for all the waves in the system ${ }^{3} S_{1}-{ }^{3} D_{1}$, and denoting by $\hat{D}_{22}(A)$ the function $D_{22}(A)$ obtained then, we have the following predictions for the subtraction constants $\delta_{3}^{(22)}$ and $\nu_{3}^{(22)}$,

$$
\begin{aligned}
\nu_{3}^{\text {pred }} & =\frac{1}{\pi} \int_{-\infty}^{L} d k^{2} \frac{\Delta_{22}\left(k^{2}\right) \hat{D}_{22}\left(k^{2}\right)}{\left(k^{2}\right)^{3}} \\
\delta_{3}^{\text {pred }} & =\frac{1}{\pi} \int_{-\infty}^{L} d k^{2} \frac{\Delta_{22}\left(k^{2}\right) \hat{D}_{22}\left(k^{2}\right)}{\left(k^{2}\right)^{2}} g_{22}^{(d)}\left(k^{2}, k_{d}^{2} ; 2\right) .
\end{aligned}
$$

The numerical values that stem from the previous expressions are $\delta_{3}^{\text {pred }} \simeq 1 m_{\pi}^{-4}$ and $\nu_{3}^{\text {pred }} \simeq-2.5 m_{\pi}^{-6}$. A fit to the ${ }^{3} D_{1}$ phase shifts only requires to vary $\nu_{3}^{(22)}$ around that value with the final result $\nu_{3}^{(22)}=$ $-2.05(5) m_{\pi}^{-6}$, while $\delta_{3}^{(22)}$ stays put. Then, it is only necessary a relatively small change of around $20 \%$ in $\nu_{3}^{(22)}$ from the one predicted by the twice-subtracted DRs in Eq. (80) in order to end with a much better reproduction of the ${ }^{3} D_{1}$ phase shifts that is compatible with the Nijmegen PWA, as shown by the hatched areas with (gray) parallel lines in Fig. 12 (denoted as NNLO-III results). Since the reproduction of the ${ }^{3} S_{1}$ phase shifts and mixing angle $\epsilon_{1}$ is the same as the one obtained already in terms of the twice-subtracted DRs, the so-called NNLO-II results, we do not show them nor the values for the other parameters given in Tables 4, 5] and 6, that would be also basically coincident with the NNLO-II ones in these tables.

We now elaborate on the difference between the value of $\nu_{3}^{(22)}$ fitted and the one predicted, $\nu_{3}^{\text {pred }}$. According to the power counting of Sec. 3.2, cf. Eq. (28),$\nu_{3}^{(22)}=\mathcal{O}\left(p^{-1}\right)$ in our present NNLO calculation. If we consider that this difference is an effect that stems from the $\mathcal{O}\left(p^{4}\right)$ contributions to $\Delta(A)$, which are not considered here yet, one would have that $\delta \nu_{3} \equiv \nu_{3}^{(22)}-\nu_{3}^{\text {pred }} \simeq 0.6 M_{\pi}^{-6}=\mathcal{O}\left(p^{0}\right)$. It also follows 
then that nominally $\delta \nu_{3} / \nu_{3}^{\text {pred }}=\mathcal{O}(p)$ and taking into account the numerical values

$$
\frac{\delta \nu_{3}}{\nu_{3}^{\text {pred }}}=0.23 \sim \frac{M_{\pi}}{\Lambda},
$$

we can estimate that $\Lambda \sim 4 M_{\pi}$, which is similar to the estimate of $\Lambda$ obtained in Sec. \for the ${ }^{1} S_{0}$ partial wave. As a result, $\delta \nu_{3}$ is consistent with a naturally sized $\mathcal{O}\left(p^{4}\right)$ effect.

The fact that the matrix of limiting values

$$
M_{i j}=\lim _{A \rightarrow-\infty} \frac{\Delta_{i j}(A)}{(-A)^{3 / 2}}
$$

has two negative eigenvalues is certainly related with the possibility of obtaining meaningful DRs with only one free parameter as first obtained in this section. We base this statement on the necessity condition of Ref. 44] in order to obtain meaningful once-subtracted DRs for $\lambda<0$, a condition also introduced in Sec. [3.2. Indeed, since the mixing between different partial waves is very small these eigenvalues are given in good approximation by $M_{11}$ and $M_{22}$; this rule applies indeed not only to the ${ }^{3} S_{1}-{ }^{3} D_{1}$ coupled waves but to any other one.

\section{Coupled ${ }^{3} P_{2}-{ }^{3} F_{2}$ waves}

We dedicate this section to the study of the coupled wave system ${ }^{3} P_{2}-{ }^{3} F_{2}$. By direct computation one has in this case that

$$
\lambda_{11}=\lim _{A \rightarrow-\infty} \frac{\Delta_{11}(A)}{(-A)^{3 / 2}}>0
$$

which requires one to consider DRs with more than one subtraction for the ${ }^{3} P_{2}$ wave [44]. Indeed, similarly to the ${ }^{3} P_{0}$ and ${ }^{3} P_{1}$ partial waves, studied in Secs. 5.1 and 5.2, respectively, we need to take at least three subtractions in the DRs for the ${ }^{3} P_{2}$ wave in order to obtain stable and meaningful results. Thus, we have the following three-time subtracted DRs for the ${ }^{3} P_{2}$ wave,

$$
\begin{aligned}
D_{11}(A) & =1+\delta_{2}^{(11)} A+\delta_{3}^{(11)} A(A-C)-\nu_{2}^{(11)} \frac{A(A-C)^{2}}{\pi} \int_{0}^{\infty} d q^{2} \frac{\nu_{11}\left(q^{2}\right)}{\left(q^{2}-A\right)\left(q^{2}-C\right)^{2}} \\
& -\nu_{3}^{(11)} \frac{A(A-C)^{2}}{\pi} \int_{0}^{\infty} d q^{2} \frac{\nu_{11}\left(q^{2}\right) q^{2}}{\left(q^{2}-A\right)\left(q^{2}-C\right)^{2}} \\
& +\frac{A(A-C)^{2}}{\pi} \int_{-\infty}^{L} d k^{2} \frac{\Delta_{11}\left(k^{2}\right) D_{11}\left(k^{2}\right)}{\left(k^{2}\right)^{3}} g_{11}\left(A, k^{2}, C ; 2\right), \\
N_{11}(A) & =\nu_{2}^{(11)} A+\nu_{3}^{(11)} A^{2}+\frac{A^{3}}{\pi} \int_{-\infty}^{L} d k^{2} \frac{\Delta_{11}\left(k^{2}\right) D_{11}\left(k^{2}\right)}{\left(k^{2}\right)^{3}\left(k^{2}-A\right)} .
\end{aligned}
$$

With respect to the mixing and ${ }^{3} F_{2}$ partial waves we use the standard formalism for the coupled waves given in Eqs. (20) and (21) with $\ell_{12}=2$ and $\ell_{22}=3$, respectively. As a result 2 and 3 subtractions are taken in order.

As usual for the $P$ waves, we fix $\nu_{2}^{(11)}=4 \pi a_{V} / m$ by requiring the exact reproduction of the ${ }^{3} P_{2}$ scattering volume extracted from the Nijmegen PWA [45],

$$
a_{V}=0.0964 M_{\pi}^{-3}
$$



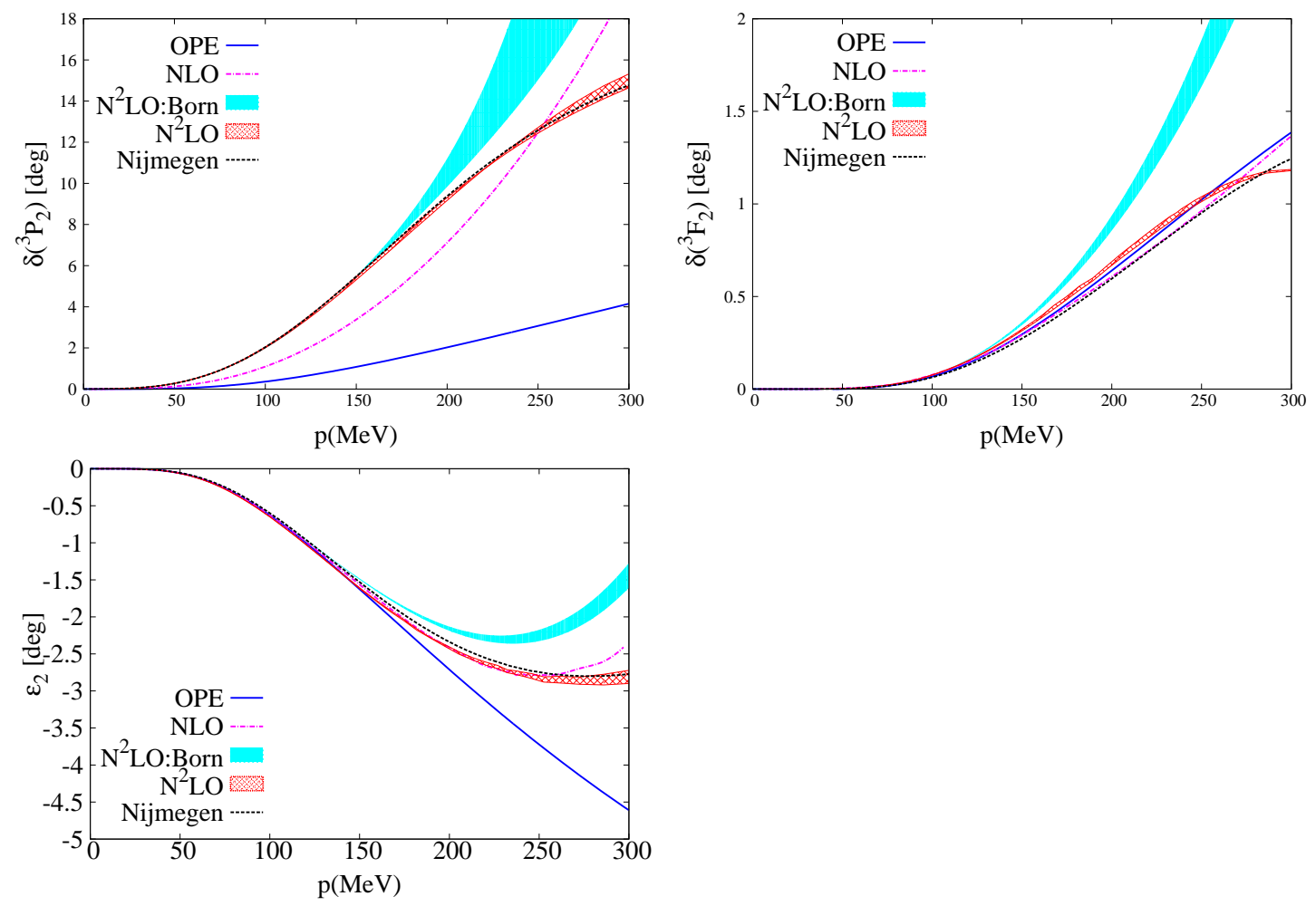

Figure 13: (Color online.) From top to bottom and left to right: Phase shifts for ${ }^{3} P_{2},{ }^{3} F_{2}$ and the mixing angle $\epsilon_{2}$, respectively. The (red) hatched areas correspond to the NNLO results and the (cyan) filled bands are the leading Born approximation results. The NLO phase shifts and mixing angle are shown by the (magenta) dot-dashed lines and the LO ones are given by the (blue) dotted lines. The Nijmegen PWA phase shifts correspond to the (black) dashed lines.

while $\nu_{3}^{(11)}$ is fitted to the results of this PWA. Regarding the subtraction constants $\delta_{i}^{(11)}, i=1,2$, we follow the principle of maximal smoothness in virtue of which we fix $\delta_{2}^{(11)}=0$ and fit $D_{11}^{(1)}\left(-M_{\pi}^{2}\right) 18$ The resulting fitted values are:

$$
\begin{aligned}
D_{11}^{(11)}\left(-M_{\pi}^{2}\right) & =0.025(5) M_{\pi}^{-2}, \\
\nu_{3}^{(11)} & =0.155(5) M_{\pi}^{-6}, \\
D_{22}^{(11)}\left(-M_{\pi}^{2}\right) & =0.011(4) M_{\pi}^{-2},
\end{aligned}
$$

with the interval of values reflecting the dependence on the $c_{i}$ 's chosen. The free parameter associated with the mixing wave is fixed to its pure perturbative value, cf. Sec. [2.3, $D_{12}\left(-M_{\pi}^{2}\right)=1$.

All in all the resulting phase shifts are shown by the (red) hatched areas in Fig. 13. There we see a clear improvement at NNLO in the reproduction of the ${ }^{3} P_{2}$ phase shifts compared with the results at NLO, given by the (magenta) dot-dashed lines, so that now the (red) hatched area overlaps the Nijmegen PWA phase shifts. The ${ }^{3} F_{2}$ phase shifts and mixing angle $\epsilon_{2}$ are reproduced with a similar quality to that already achieved at NLO. We also give by the (cyan) filled bands the results obtained by the leading

\footnotetext{
${ }^{18}$ In the following we use $D_{i j}^{p-2}\left(-M_{\pi}^{2}\right)$ as free parameter in terms of which one can calculate $\delta_{p}^{(i j)}$ from Eq. (22).
} 
Born approximation, Eq. (58), with $\Delta(A)$ calculated at NNLO. Due to the fact that the latter diverges as $(-A)^{3 / 2}$ for $A \rightarrow-\infty$ at least two subtractions have to be taken in the DR for $N_{B}(A)$, Eq. (57). This is immediately accomplished for the $D$ and higher partial waves but for a $P$-wave with $\ell=1$ one needs to include one extra subtraction. In particular, for our present case we use Eq. (85) with $D_{11}(A) \rightarrow 1$ and with $\Delta_{11}\left(k^{2}\right)$ restricted to its two-nucleon irreducible contributions, with the subtraction constants $\nu_{2}^{(11)}$ and $\nu_{3}^{(11)}$ taking the same values as discussed before. We see that our full results provide a clear improvement in the reproduction of the Nijmegen PWA phase shifts and mixing angle with respect to the Born approximation. One should mention that the Born approximation phase shifts for ${ }^{3} F_{2}$ and ${ }^{3} F_{3}$ have a striking resemblance to the full NNLO results of Ref. [32] obtained within the Weinberg scheme. We have obtained this improvement without dismissing the strength of the TPE at NNLO, as advocated in Ref. [48]. This makes that our full results are not so much sensitive to the particular set of $c_{i}$ 's taken as previously thought in the literature from the results of Refs. [32,48].

\section{Coupled ${ }^{3} D_{3}-{ }^{3} G_{3}$ waves}
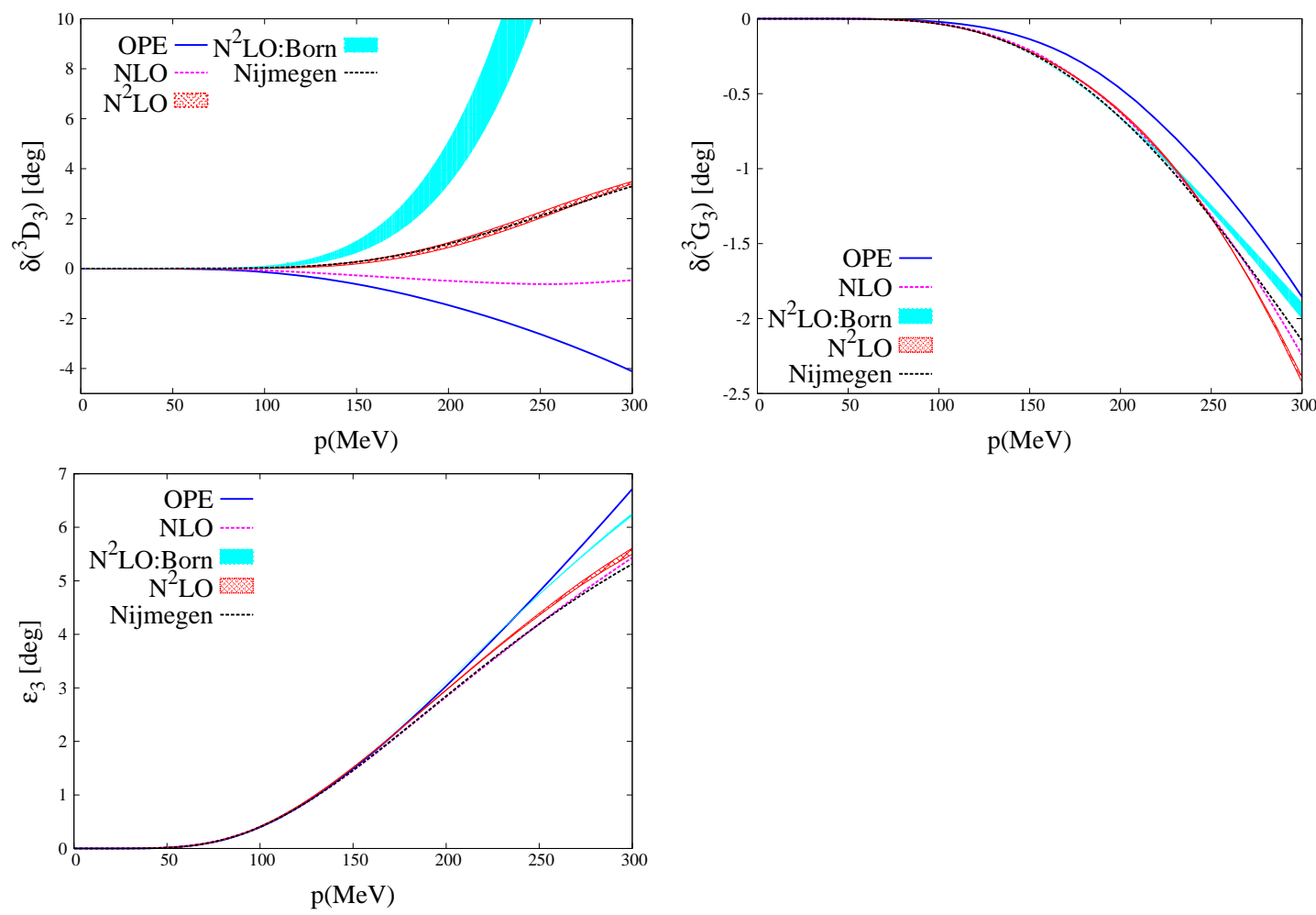

Figure 14: (Color online.) From top to bottom and left to right: Phase shifts for ${ }^{3} D_{3},{ }^{3} G_{3}$ and the mixing angle $\epsilon_{3}$, in order. The (red) hatched areas correspond to the NNLO results and the (cyan) filled bands are the leading order Born approximation. The NLO results are shown by the (magenta) dot-dashed lines and the LO ones are given by the (blue) dotted lines. The Nijmegen PWA phase shifts correspond to the (black) dashed lines.

For the study of the ${ }^{3} D_{3}-{ }^{3} G_{3}$ coupled waves we follow the formalism for coupled waves, Eqs. (20) and (21), with $\ell_{11}=2, \ell_{12}=3$ and $\ell_{22}=4$, so that $\ell_{i j}$ subtractions are taken in the DRs for the coupled 
wave $i j$. Regarding the free parameters we follow the principle of maximal smoothness, although for the mixing wave the subtraction constants take their pure perturbative values. So that we fit to data $D_{11}\left(-M_{\pi}^{2}\right)$ and $D_{22}^{(2)}\left(-M_{\pi}^{2}\right)$, with the resulting values:

$$
\begin{aligned}
D_{11}\left(-M_{\pi}^{2}\right) & =0.90(5), \\
D_{22}^{(2)}\left(-M_{\pi}^{2}\right) & =-0.09(1) M_{\pi}^{-4},
\end{aligned}
$$

The interval of values in Eq. (89) reflect the dependence on the set of values considered for the $c_{i}$ 's. The resulting phase shifts are shown by the (red) hatched areas in Fig. 14. Importantly at NNLO the phase shifts for the ${ }^{3} D_{3}$ wave follow closely the Nijmegen PWA phase shifts so that a remarkable improvement is obtained in comparison with both the NLO and Born results. Notice that this is accomplished without any need of dismissing the strength of TPE as directly obtained from the NLO $\pi N$ amplitudes. We have been able to improve the situation by taking into account the subtraction constant $\delta_{2}$ or $D_{11}\left(-M_{\pi}^{2}\right)$, whose presence is required by the nonperturbative unitarity implementation 19 at NNLO, cf. Eq. (28). We also observe a good reproduction of the Nijmegen PWA results for the waves ${ }^{3} G_{3}$ and $\epsilon_{3}$, which are already well reproduced at NLO [44] as shown by the (magenta) dot-dashed lines.

\section{Coupled ${ }^{3} F_{4}-{ }^{3} H_{4}$ waves}

The discussion of the ${ }^{3} F_{4}-{ }^{3} H_{4}$ coupled-wave system follows the standard formalism for coupled waves, Eq. (20) and (21), with $\ell_{11}=3, \ell_{12}=4$ and $\ell_{22}=5$. The free parameters are then fitted to data according to the principle of maximal smoothness. However, for ${ }^{3} H_{4}$ and the mixing partial wave there is no improvement in the reproduction of data with respect to the situation in which the pure perturbative values are taken, so that at the end we only have to fit $D_{11}^{(1)}\left(-M_{\pi}^{2}\right)$ to the Nijmegen PWA results. The fitted value is

$$
D_{11}^{(1)}\left(-M_{\pi}^{2}\right)=-0.009(3) M_{\pi}^{-2} .
$$

The resulting phase shifts and mixing angle are shown by the (red) hatched areas in Fig [15, with the width of the band reflecting the dependence on values for the $\pi N$ NLO counterterms. One can observe a clear improvement in the description of the ${ }^{3} F_{4}$ phase shifts compared with the results from OPE (blue dotted lines), NLO (magenta dot-dashed lines) and leading Born approximation (cyan filled areas). Similarly to the ${ }^{3} D_{3}$ wave in the previous section, this improvement is related with the effect of the subtraction constant $\delta_{3}^{(11)}$ which is not directly related with an improvement in the calculation of $\Delta_{11}(A)$, and hence of the $N N$ potential. Let us recall that the subtraction constants $\delta_{p}^{(i j)}$ arise because of the rescattering process that the $N / D$ method allows to treat in a clear and well-defined way, overcoming the obscurities that still remain in the literature associated with the use of the cutoff regularized Lippmann-Schwinger with a higher-order $N N$ potential. For the mixing angle $\epsilon_{4}$ the quality in the reproduction of data is similar to that obtained by the other approximations just quoted. However, for the ${ }^{3} H_{4}$ phase shifts the outcome at NNLO is a bit worse than at NLO and OPE, though one should also notice the tiny values for the ${ }^{3} H_{4}$ phase shifts so that this discrepancy is certainly small in absolute value. We have also checked that it cannot be removed by releasing the other subtraction constants $\delta_{p}^{(22)}$, with $p=2,3$ and 4 . Likely, the origin of this difference in the ${ }^{3} \mathrm{H}_{4}$ phase shifts between our full results and the Nijmegen PWA can be tracked back to the change in the leading Born approximation once the $\mathcal{O}\left(p^{3}\right)$ two-nucleon irreducible contributions are included in $\Delta_{22}(A)$.

\footnotetext{
${ }^{19}$ In more general terms, by generating the analytical properties associated with the RHC while respecting unitarity in the full amplitudes.
} 

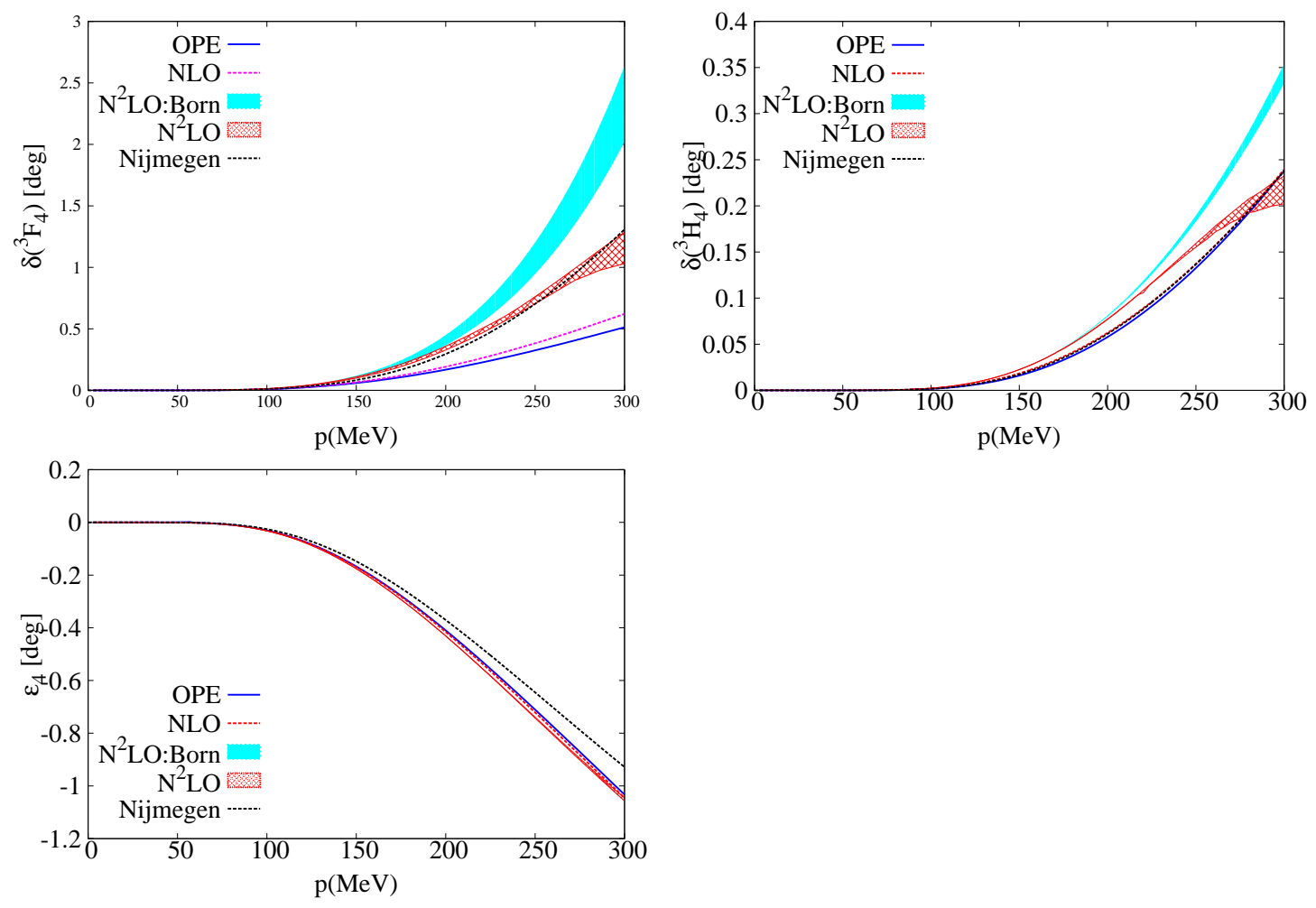

Figure 15: (Color online.) From top to bottom and left to right: Phase shifts for ${ }^{3} F_{4},{ }^{3} H_{4}$ and the mixing angle $\epsilon_{4}$, in order. The (red) hatched areas correspond to the NNLO results and the (cyan) filled ones to the leading Born approximation. The NLO results are shown by the (magenta) solid line and the LO ones are given by the (blue) dotted lines. The Nijmegen PWA phase shifts are given by the (black) dashed lines.

\section{Coupled ${ }^{3} G_{5}-{ }^{3} I_{5}$ waves}

The standard formalism for coupled waves with high angular momentum, Eqs. (20) and (21), is followed here with $\ell_{11}=4, \ell_{12}=5$ and $\ell_{22}=6$. The application of the principle of maximal smoothness to fit the free parameters provides a good reproduction of the Nijmegen PWA phase shifts [45],20] The range of values obtained for the free parameters $D_{11}^{(2)}\left(-M_{\pi}^{2}\right)$ and $D_{22}^{4}\left(-M_{\pi}^{2}\right)$ is

$$
\begin{aligned}
& D_{11}^{(2)}\left(-M_{\pi}^{2}\right)=-0.0025(5) M_{\pi}^{-4}, \\
& D_{22}^{(4)}\left(-M_{\pi}^{2}\right)=-0.0125(5) M_{\pi}^{-8},
\end{aligned}
$$

while basically the same results are obtained for any $D_{12}^{(3)}\left(-M_{\pi}^{2}\right) \leq 0 M_{\pi}^{-6}$. The results are shown in Fig 16 by the (red) hatched areas whose widths take into account the uncertainty from the set of $c_{i}$ 's taken and some numerical noise from the iterative process. A clear improvement results in the description of the ${ }^{3} G_{5}$ phase shifts compared with the OPE (blue dotted lines), NLO (magenta dot-dashed lines) and leading Born approximation results (cyan filled areas). It is worth stressing that this partial wave cannot be well reproduced even at NNNLO in the Weinberg potential scheme neither by keeping a finite value

\footnotetext{
${ }^{20}$ For $5 \leq J \leq 8$ the Nijmegen PWA phase shifts [45] are those obtained from the $N N$ potential model of Ref. 64.
} 

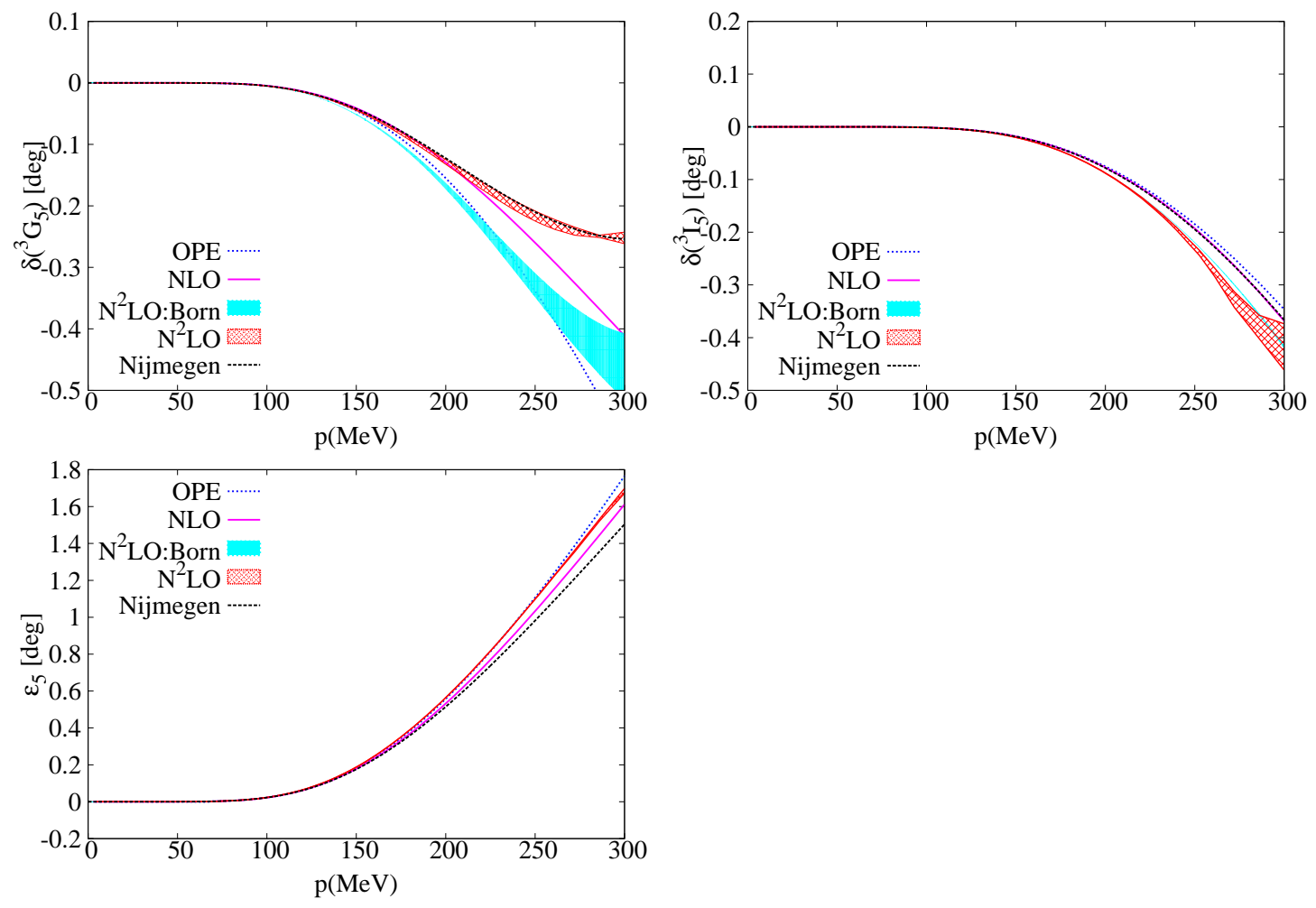

Figure 16: (Color online.) From top to bottom and left to right: Phase shifts for ${ }^{3} G_{5},{ }^{3} I_{5}$ and the mixing angle $\epsilon_{5}$, in order. The (red) hatched areas correspond to the NNLO results and the filled ones to the leading Born approximation. The NLO results are shown by the (magenta) dot-dashed line and the LO ones are given by the (blue) dotted lines. The Nijmegen PWA phase shifts are shown by the (black) dashed lines.

for the three-momentum cutoff entering in the solution of the Lippmann-Schwinger equation [33], nor by sending it to $\infty$ as in Ref. [40. A similar situation occurs too for the leading Born approximation results at NNLO, as shown by the (cyan) filled area in the first panel, a result also obtained in Ref. [48. Even more, the modification of the TPE mechanism proposed in this reference by making use of the so-called spectral-function regularization is inoperative here to provide an improvement in the Born approximation results. A similar problem was also observed in the perturbative calculation at NNNLO in Ref. [31]. From ours results this is not surprising because the improvement in the reproduction of the Nijmegen PWA phase shifts for the ${ }^{3} G_{5}$ wave is accomplished through the subtraction constant $\delta_{4}^{(11)}$. This constant is directly related to the $N N$ rescattering (from which the final function $D_{11}(A)$ stems nonperturbatively) and not to the $N N$ potential or $\Delta_{22}(A)$. In the case of the mixing angle $\epsilon_{5}$ and the ${ }^{3} I_{5}$ phase shifts there is a slight worsening in the reproduction of Nijmegen PWA compared with the NLO ones, but still our results run very close to the Nijmegen PWA ones.

Finally, we give in Table 7 the values of the free parameters employed in the different partial waves according to the type of DRs employed, which is indicated in the second column. This is done by following the notation, already introduced in Ref. [44, $m \mathrm{DR}$ with $m=1,2, \ldots$, and it should be read as $m$-time subtracted DR. For the higher $N N$ partial waves we use the abbreviation LTS to indicate that $\ell$ (or $J$ for the mixing partial waves) subtractions are taken to satisfy the threshold behavior, following the standard 


\begin{tabular}{|l|l|l|}
\hline Wave & Type of DRs & Parameters \\
\hline${ }^{1} S_{0}$ & 1 DR & $\nu_{1}=30.69$ \\
& 2 DR & $\nu_{1}=30.69, \nu_{2}=-23(1), \delta_{2}=-8.0(3)$ \\
\hline${ }^{3} P_{0}$ & 3 DR & $\nu_{2}=1.644, \delta_{2}=2.82(5), \delta_{3}=0.18(6)$ \\
\hline${ }^{3} P_{1}$ & 3 DR & $\nu_{2}=-1.003, \delta_{2}=2.7(1), \delta_{3}=0.47(3)$ \\
\hline${ }^{1} P_{1}$ & 2 DR & $\nu_{2}=-1.723, \delta_{2}=0.4(1)$ \\
\hline${ }^{1} D_{2}$ & LTS & $D^{(1)}(0)=0.07(1)$ \\
\hline${ }^{3} D_{2}$ & LTS & $D^{(1)}(0)=-0.017(3)$ \\
\hline${ }^{1} F_{3}$ & LTS & $D^{(2)}(0)=0.057(3)$ \\
\hline${ }^{3} F_{3}$ & LTS & $D^{(2)}(0)=0.035(5)$ \\
\hline${ }^{1} G_{4}$ & LTS & $D^{(3)}(0)=-0.014(2)$ \\
\hline${ }^{3} G_{4}$ & LTS & $D^{(3)}(0)=-0.055(5)$ \\
\hline${ }^{1} H_{5}$ & LTS & $D^{(4)}(0)=0.156$ \\
\hline${ }^{3} H_{5}$ & LTS & $D^{(4)}(0)=0.066$ \\
\hline${ }^{3} S_{1}-{ }^{3} D_{1}$ & 1 DR ${ }^{3} S_{1}, 2$ DR ${ }^{3} D_{1}$, mixing & $E_{d}$ \\
& 2DR all & $a_{t}, r_{t}, E_{d}$ \\
& DR ${ }^{3} S_{1}$, mixing, 3 DR ${ }^{3} D_{1}$ & $a_{t}, r_{t}, E_{d}, \nu_{3}^{(22)}=-2.05(5)$ \\
\hline${ }^{3} P_{2}-{ }^{3} F_{2}$ & 3 DR for ${ }^{3} P_{2}$ and LTS for the others & $\nu_{2}^{(11)}=0.178, D_{11}^{(1)}\left(-M_{\pi}^{2}\right)=0.025(5), \nu_{3}^{(11)}=0.155(5)$ \\
& & $D_{22}\left(-M_{\pi}^{2}\right)=0.011(4)$ \\
\hline${ }^{3} D_{3}-{ }^{3} G_{3}$ & LTS & $D_{11}\left(-M_{\pi}^{2}\right)=0.90(5), D_{22}^{(2)}\left(-M_{\pi}^{2}\right)=-0.09(1)$ \\
\hline${ }^{3} F_{4}-{ }^{3} H_{4}$ & LTS & $D_{11}^{(1)}\left(-M_{\pi}^{2}\right)=-0.009(3)$ \\
\hline${ }^{3} G_{5}-{ }^{3} I_{5}$ & LTS & $D_{11}^{(2)}\left(-M_{\pi}^{2}\right)=-0.0025(5), D_{22}^{(4)}\left(-M_{\pi}^{2}\right)=-0.0125(5)$ \\
\hline
\end{tabular}

Table 7: We give in the columns from left to right, in order, the partial wave, the type of DRs employed to study it and the values for the free parameters involved. 
formalism explained in Sec. 2.3. According to the principle of maximal smoothness only the highest derivative $D^{(n)}(C)$ related to the subtraction constants in $D(A)$ is not fixed to its perturbative value (1 for $n=0$ and 0 for $n \neq 0$ ) and released, if appropriate. The units correspond to appropriate powers of $M_{\pi}^{2}$, although they are not explicitly shown. There is a proliferation of free parameters for the $P$ waves because for them $\lambda>0$, Eqs. (45) and (83), so that, except for the ${ }^{1} P_{1}$ wave, three-time-subtracted DRs are needed. This could be a specific feature for the NNLO calculation of $\Delta(A)$ that has to be investigated for higher-orders 21

\section{Conclusions}

We have discussed in this paper the application of the $N / D$ method when its dynamical input, namely, the imaginary part of the $N N$ partial waves along the LHC, is calculated in ChPT up to NNLO. It then comprises OPE, leading and subleading two-nucleon irreducible TPE and once-iterated OPE [46]. We have obtained a quite good reproduction of the Nijmegen PWA phase shifts and mixing angles, in better agreement than the one achieved in the previous lower order studies at LO [42, 43] and NLO [44]. In particular, our NNLO results are able to reproduce the phase shifts for the triplet waves with $\ell_{11}=J-1$, ${ }^{3} P_{2},{ }^{3} D_{3},{ }^{3} F_{4}$ and ${ }^{3} G_{5}$, while at NLO they were not properly accounted for. We do not need to modify the NNLO two-nucleon irreducible diagrams (or chiral $N N$ potential) in order to obtain such a good agreement with the Nijmegen PWA, contrary to common wisdom. The point that stems from our study is that one should perform in a well-defined way the iteration of diagrams along the RHC, which are responsible for unitarity and analyticity attached to this cut, rather than reshuffling the $N N$ potential with contributions from higher orders. In this respect, the use of DRs allows one to perform the iteration of two-nucleon intermediate states independently of regulator. We have also compared our full results for the higher partial waves with the Born approximation. From this comparison, as well as from the direct study of the importance of the different contributions of $\Delta(A)$ to the dispersive integrals, it follows that the $N N D$ waves cannot be treated perturbatively.

It is also worth remarking that up to the order studied here we reproduce the long-range correlation between the effective ranges and the scattering lengths for the $N N S$ waves when only once-subtracted DRs are applied. In this way one can predict values for the $S$-wave effective ranges in agreement with experiment up to around a $10 \%$. We have also elaborated a chiral power counting for the subtraction constants, so that twice-subtracted DRs are appropriate when $\Delta(A)$ is calculated at NLO and NNLO. From these considerations it turns out also that the chiral power expansion is made over a scale $\Lambda \sim$ $400 \mathrm{MeV}$. One should consider further the impact of higher orders in $\Delta(A)$, which are partially calculated already in the literature, as an interesting extension of the present work in order to settle the applicability of the $N / D$ method to $N N$ scattering in ChPT with a high degree of accurateness.

\section{Acknowledgments}

This work is partially funded by the grants MINECO (Spain) and ERDF (EU), grant FPA2010-17806 and the Fundación Séneca 11871/PI/09. We also thank the financial support from the EU-Research Infrastructure Integrating Activity "Study of Strongly Interacting Matter" (HadronPhysics2, grant n. 227431) under the Seventh Framework Program of EU and the Consolider-Ingenio 2010 Programme CPAN (CSD2007-00042).

\footnotetext{
${ }^{21}$ If then $\lambda<0$ one would need to invoke less free parameters for the $P$ waves than in Table 7
} 


\section{A Calculation of higher order shape parameters}

Let us explain first the method for the ${ }^{1} S_{0}$ partial wave, which is then straightforwardly generalized to the ${ }^{3} S_{1}$ case. Taking into account Eq. (5) we have that

$$
H(A) \equiv \frac{4 \pi}{m T(A)}+i \sqrt{A}=\sqrt{A} \cot \delta
$$

is an analytical function of $A$ that has no (elastic) unitarity cut because it obeys the Schwarz reflection principle and it is real for $A>0$. Then it admits a Taylor expansion around $A=0$ with a radius of convergence equal to $M_{\pi}^{2} / 4$, since its first singularity is due to the onset of the LHC at $A=-M_{\pi}^{2} / 4$. This expansion is the so-called ERE.

We can calculate the function $H(A)$ for complex $A$ in a direct way from the DRs of Eqs. (29), (30), for the once-subtracted case, and from Eqs. (38) and (39) in terms of twice-subtracted DRs. Nonetheless, care has to be taken when employing $g\left(A, k^{2}\right)$ from Eq. (9) because one should guarantee that $\sqrt{A}$ is defined in the first Riemann sheet, that is, $\operatorname{Im} \sqrt{A}>0$ must be enforced for all $A \in \mathbb{C}$. The same requirement should be also fulfilled by the $\sqrt{A}$ that appears explicitly in the definition of $H(A)$.

The $n$th order derivative of $H(A)$ at $A=0$ can be calculated by making use of the Cauchy's integral formula

$$
H^{(n)}(0)=\frac{n !}{2 \pi i} \oint_{\gamma} d z \frac{H(z)}{z^{n+1}}
$$

where $\gamma$ is a close contour inside the ball of radius $M_{\pi}^{2} / 4$ and taken counter-clockwise. In practical terms we take the contour $\gamma$ as a circle of radius $R<M_{\pi}^{2} / 4$ with $z=R \exp i \phi$ and $\phi \in[0,2 \pi]$. A good numerical check of the procedure is the stability of the derivative calculated from the previous equation independently of the value taken for $0<R<M_{\pi}^{2} / 4$. Thus, we obtain

$$
\begin{aligned}
a_{s}^{-1} & =-\frac{1}{2 i \pi} \oint d z \frac{H(z)}{z}, \\
r_{s} & =\frac{1}{i \pi} \oint d z \frac{H(z)}{z^{2}}, \\
v_{i} & =\frac{1}{2 i \pi} \oint d z \frac{H(z)}{z^{i+1}} .
\end{aligned}
$$

We can proceed in the same way for the ${ }^{3} S_{1}-{ }^{3} D_{1}$ coupled wave system in terms of the eigenvalue $S_{0}$ given by

$$
S_{0}=\frac{1}{2}\left[S_{11}+S_{22}+\left(S_{11}-S_{22}\right) \sqrt{1+\left(\frac{2 S_{12}}{S_{11}-S_{22}}\right)^{2}}\right] .
$$

Then, we define in terms of it the corresponding uncoupled partial wave

$$
T_{0}(A)=\frac{S_{0}-1}{2 i \rho(A)}
$$

where the definition of $\rho(A)$ in Eq. (3) should be taken in the first Riemann sheet. An analogous function to $H(A)$ in Eq. (A.1) is then constructed from $T_{0}(A)$ and we can calculate the different parameters in the ERE of Eq. (75) as in Eq. (A.3). 


\section{References}

[1] S. Weinberg, Physica A 96 (1979) 327.

[2] J. Gasser and H. Leutwyler, Ann. Phys. 158 (1984) 142; Nucl. Phys. B 250 (1985) 465.

[3] J. A. Oller and E. Oset, Nucl. Phys. A 620 (1997) 438; (E) ibid. A 652 (1999) 407.

[4] J. A. Oller and E. Oset, Phys. Rev. D 60 (1999) 074023.

[5] J. A. Oller and L. Roca, Phys. Lett. B 651 (2007) 139.

[6] J. A. Oller, L. Roca and C. Schat, Phys. Lett. B 659 (2008) 201.

[7] M. Albaladejo and J. A. Oller, Phys. Rev. D 86 (2012) 034003.

[8] B. Ananthanarayan, G. Colangelo, J. Gasser and H. Leutwyler, Phys. Rep. 353 (2001) 207.

[9] T. N. Truong, Phys. Rev. Lett. 61 (1988) 2526.

[10] J. Gasser, H. Leutwyler and M. E. Sainio, Phys. Lett. B 253 (1991) 252; Phys. Lett. B 253 (1991) 260.

[11] J. M. Alarcon, J. M. Camalich and J. A. Oller, Annals Phys. 336 (2013) 413.

[12] J. M. Alarcon, J. Martin Camalich and J. A. Oller, Phys. Rev. D 85 (2012) 051503.

[13] J. Gasser, M. E. Sainio and A. Svarc, Nucl. Phys. B 307 (1988) 779.

[14] E. E. Jenkins and A. V. Manohar, Phys. Lett. B 255 (1991) 558.

[15] J. M. Alarcon, L. S. Geng, J. Martin Camalich and J. A. Oller, ev arXiv:1209.2870 [hep-ph]. To be published in Phys. Lett. B.

[16] J. Gegelia and G. Japaridze, Phys. Rev. D 60 (1999) 114038; T. Fuchs, J. Gegelia, G. Japaridze and S. Scherer, Phys. Rev. D 68 (2003) 056005.

[17] V. Pascalutsa, M. Vanderhaegen and S. N. Yang, Phys. Rep. 437 (2007) 125; V. Pascalutsa and D. R. Phillips, Phys. Rev. C 67 (2003) 055202.

[18] J. Bijnens, PoS CD 09 (2009) 031; Prog. Part. Nucl. Phys. 58 (2007) 521.

[19] G. Ecker, Nucl. Phys. Proc. Suppl. 245 (2013) 1; Prog. Part. Nucl. Phys. 35 (1995) 1.

[20] V. Bernard, Prog. Part. Nucl. Phys. 60 (2008) 82.

[21] A. Pich, Rep. Prog. Phys. 58 (1995) 563; Proc. of Les Houches Summer School of Theoretical Physics, Vol. II, F. David and R. Gupta, eds., Elsevier Science B.V., Amsterdam, 1999 hep-ph/9806303].

[22] V. Bernard, N. Kaiser and U.-G. Meißner, Int. J. Mod. Phys. E 4 (1995) 193; Rep. Prog. Phys. 56 (1993) 903.

[23] S. Weinberg, Phys. Lett. B 251 (1990) 288; Nucl. Phys. B 363 (1991) 3. 
[24] J. A. Oller, A. Lacour and U.-G. Meißner, J. Phys. G 37 (2010) 015106; A. Lacour, J. A. Oller and U.-G. Meißner, Annals Phys. 326 (2011) 241.

[25] E. Epelbaum, H. -W. Hammer and U.-G. Meißner, Rev. Mod. Phys. 81 (2009) 1773.

[26] R. Machleidt and D. R. Entem, Phys. Rept. 503 (2011) 1

[27] E. Epelbaum, Prog. Part. Nucl. Phys. 57 (2006) 654.

[28] P. F. Bedaque and U. van Kolck, Ann. Rev. Nucl. Part. Sci. 52 (2002) 339.

[29] U. van Kolck, Prog. Part. Nucl. Phys. 43 (1999) 337.

[30] C. Ordóñez, L. Ray and U. van Kolck, Phys. Lett. B 291 (1992) 459; Phys. Rev. Lett. 72 (1994) 1982; Phys. Rev. C 53 (1996) 2086.

[31] D. R. Entem and R. Machleidt, Phys. Lett. B 524 (2002) 93; Phys. Rev. C 66 (2002) 014002; Phys. Rev. C 68 (2003) 041001.

[32] E. Epelbaum, PhD thesis, published in Berichte des Forschungszentrum Jülich, No. 3803 (2000); E. Epelbaum, W. Gloeckle and U.-G. Meißner, Nucl. Phys. A 671 (2000) 295 .

[33] E. Epelbaum, W. Gloeckle and U.-G. Meißner, Nucl. Phys. A 747 (2005) 362.

[34] M. Pavón Valderrama and E. Ruiz Arriola, Phys. Rev. C 74 (2006) 054001; ibid 74 (2006) 064004; ibid 72 (2005) 054002.

[35] A. Nogga, R. G. E. Timmermans and U. van Kolck, Phys. Rev. C 72 (2005) 054006.

[36] D. B. Kaplan and M. J. Savage, Phys. Lett. B 365 (1996) 244; D. B. Kaplan, M. J. Savage and M. B. Wise, Nucl. Phys. B 478 (1996) 629.

[37] C.-J. Yang, C. Elster and D. R. Phillips, Phys. Rev. C 77 (2008) 014002; idem 80 (2009) 034002; idem 80 (2009) 044002.

[38] M. Pavon Valderrama, Phys. Rev. C 84 (2011) 064002.

[39] B. Long and C. J. Yang, Phys. Rev. C 84 (2011) 057001; ibid 85 (2012) 034002; ibid 86 (2012) 024001.

[40] C. Zeoli, R. Machleidt and D. R. Entem, Few Body Syst. 54 (2013) 2191.

[41] G. F. Chew and S. Mandelstam, Phys. Rev. 119 (1960) 467.

[42] M. Albaladejo and J. A. Oller, Phys. Rev. C 84 (2011) 054009.

[43] M. Albaladejo and J. A. Oller, Phys. Rev. C 86 (2012) 034005.

[44] Z.-H. Guo, J. A. Oller and G. Ríos, Phys. Rev. C 89 (2014) 014002.

[45] V. G. J. Stoks, R. A. M. Klomp, C. P. F. Terheggen and J. J. de Swart, Phys. Rev. C49 (1994) 2950.

[46] N. Kaiser, R. Brockmann and W. Weise, Nucl. Phys. A 625 (1997) 758. 
[47] A. M. Gasparyan, M. F. M. Lutz and E. Epelbaum, Eur. Phys. J. A 49 (2013) 115.

[48] E. Epelbaum, W. Gloeckle and U.-G. Meißner, Eur. Phys. J. A 19 (2004) 125; Eur. Phys. J. A 19 (2004) 401.

[49] G. Barton, "Introduction to Dispersion Techniques in Field Theory", W. A. Benjamin, Inc., New York, 1965.

[50] M. Sugawara and A. Kanazawa, Phys. Rev. 123 (1961) 1895.

[51] A. D. Martin and T. D. Spearman, "Elementary Particle Theory", North-Holland Publishing Company, Amsterdam. 1970.

[52] H. P. Stapp, T. J. Ypsilantis and N. Metropolis, Phys. Rev. 105 (1957) 302.

[53] R. Koch, Nucl. Phys. A 448, 707 (1986).

[54] R. A. Arndt et al., Phys. Rev. C 74, 045205 (2006) and references therein; Computer code SAID, online program at http://gwdac.phys.gwu.edu/

[55] H. Krebs, A. Gasparyan and E. Epelbaum, Phys. Rev. C 85 (2012) 054006.

[56] P. Buettiker and U.-G. Meißner, Nucl. Phys. A 668 (2000) 97.

[57] J. M. Alarcon, J. Martin Camalich, J. A. Oller and L. Alvarez-Ruso, Phys. Rev. C 83 (2011) 055205.

[58] M. C. M. Rentmeester, R. G. E. Timmermans and J. J. de Swart, Phys. Rev. C 67, 044001 (2003).

[59] N. Fettes and U.-G. Meißner, Nucl. Phys. A 676 (2000) 311; N. Fettes, U.-G. Meißner and S. Steininger, Nucl. Phys. A 640 (1998) 199.

[60] V. V. Flambaum, G. F. Gribakin and C. Harabati, Phys. Rev. A 59 (1999) 1998; G. F. Gribakin and V. V. Flambaum, Phys. Rev. A 58 (1993) 546.

[61] A. Calle Cordon and E. Ruiz Arriola, Phys. Rev. A 81 (2010) 044701.

[62] V. Bernard, N. Kaiser and U.-G. Meißner, Phys. Lett. B 309, 421 (1993).

[63] J. M. Alarcon, J. Martin Camalich and J. A. Oller, Phys. Rev. D 85, 051503 (2012).

[64] M. M Nagels, T. A. Rijken and J. J. de Swart, Phys. Rev. D 17 (1978) 768.

[65] J. J. de Swart, C. P. F. Terheggen and V. G. J. Stoks, Proceedings of 3rd International Symposium on Dubna Deuteron 95, Dubna, Moscow, July 4-7, 1995, arXiv: nucl-th/9509032.

[66] T. D. Cohen and J. M. Hansen, Phys. Rev. C 59 (1999) 13.

[67] N. L. Rodning and L. D. Knutson, Phys. Rev. C 41 (1990) 898.

[68] T. E. O. Ericson and M. Rosa-Clot, Phys. Lett. B 110 (1982) 193; Nucl. Phys. A 405 (1983) 497.

[69] H. E. Conzett, F. Hinterberger, P. von Rossen, F. Seiler and E. J. Stephenson, Phys. Rev. Lett. 43 (1979) 572.

[70] S. Klarsfeld, J. Martorell and D. W. L. Sprung, J. Phys. G: Nucl. Phys. 10 (1984) 165. 